\title{
Un manuscrito de finales del siglo XVII: primera manifestación de un estudio astronómico y cronológico autóctono en territorio neogranadino
}

\author{
J. Gregorio Portilla ${ }^{1} \&$ Freddy Moreno ${ }^{2}$ \\ ${ }^{1}$ Observatorio Astronómico Nacional, Facultad de Ciencias, Sede Bogotá, Universidad Nacional de Colombia \\ ${ }^{2}$ Centro de Estudios Astrofísicos, colegio Gimnasio Campestre
}

\begin{abstract}
Resumen
La existencia de un manuscrito que versa sobre astronomía y cronología redactado a finales del siglo XVII por Antonio Sánchez de Cozar, un humilde sacerdote de ascendencia indígena, ha pasado desapercibida para los historiadores de la ciencia en el país, aun cuando ya a mediados del siglo XIX había sido dado a conocer como pieza literaria. En este trabajo se presenta una síntesis del contenido de ese manuscrito, así como un análisis de algunos aspectos relevantes que muestran un pensamiento original e independiente del autor, tales como su propuesta dinámica del movimiento de los cielos, la introducción de un cielo entre Mercurio y la Luna y la propuesta de reforma al calendario gregoriano. El manuscrito también contiene las primeras efemérides astronómicas realizadas en territorio neogranadino, consistentes en fases lunares y eclipses cuyos tiempos de ocurrencia están referidos a la hora local de una población del Nuevo Mundo: el municipio de Vélez. C2019. Acad. Colomb. Cienc. Ex. Fis. Nat.
\end{abstract}

Palabras clave: modelo geocéntrico; calendario; cronología; efemérides; eclipses.

A manuscript of the late seventeenth century: first manifestation of an astronomical and chronological autochthonous study in the Nueva Granada

\begin{abstract}
The existence of a manuscript that deals with astronomy and chronology written in the late seventeenth century by Antonio Sánchez de Cozar, a humble priest of indigenous descent, has gone unnoticed by historians of science in the country even though in the mid-nineteenth century it had been given to know as a literary work. In this paper a synthesis of the content of this manuscript is presented, as well as an analysis of some relevant aspects that show an original and independent thought of the author, such as his cinematic proposal of the movement of the heavens, the introduction of a sky between Mercury and the Moon and the proposed reform of the Gregorian calendar. The manuscript also con tains the first astronomical ephemerides made in Neo-Granadian territory consisting of lunar phases and eclipses whose times of occurrence are referred to the local time of a town of the New World: the municipality of Vélez. (C2018. Acad. Colomb. Cienc. Ex. Fis. Nat.
\end{abstract}

Key words: Geocentric model; calendar; chronology; ephemerides: eclipses.

\section{Introducción}

Es poco lo que se conoce sobre la realización de estudios astronómicos en el periodo colonial español en lo que es ahora territorio colombiano. Sin embargo, varios trabajos relativamente recientes han permitido mostrar que en Santafé de Bogotá, específicamente en el Colegio San Bartolomé, se impartieron lecciones en las que se llegaron a exponer temas de naturaleza astronómica. Es así como, a finales del siglo XVII, se redactó un documento titulado "Breve tratado del cielo y de los astros", el cual es un complemento a un manuscrito de nombre "Tratado de Física" cuyo autor es el sacerdote jesuita Mateo Mimbela (1663-1736) (Del Rey \& Marquínez, 2004). Así mismo, medio siglo después, en 1755, otro sacerdote jesuita, Francisco Javier Trías (1721¿?), redactó un documento que lleva por título "Física especial y curiosa” (Marquínez \& Del Rey, 2005).

\footnotetext{
Correspondencia:

J. Gregorio Portilla, jgportillab@unal.edu.co

Recibido: 1 de mayo de 2019
}

Aceptado: 8 de junio de 2019

Editora: Elizabeth Castañeda 
Los dos documentos tienen varios aspectos en común: fueron redactados en latín, sus autores son sacerdotes nacidos en España y no pasan de ser apuntes de clase destinados a una de las cátedras que se impartieron en el colegio San Bartolomé. El objeto de estos manuscritos es simplemente la transmisión de conocimiento y no se advierte en ellos ni originalidad ni algún fruto proveniente de un proceso investigativo. El primer documento desglosa la teoría geocéntrica del universo dentro de un contexto claramente escolástico; se cita a Copérnico, Brahe y Galilei, aunque sea para oponerse al modelo heliocéntrico, y se evidencia que su autor está al tanto de los primeros descubrimientos obtenidos a través del telescopio. El segundo documento, evidentemente más moderno, expone los tres sistemas del mundo (el de Ptolomeo, el de Copérnico y de Brahe) y se muestra contrario al sistema geocéntrico. Esto último indica que ya se había ofrecido al menos una cátedra en el virreinato de la Nueva Granada en la que se dio a conocer el heliocentrismo antes de las célebres conferencias ofrecidas por José Celestino Mutis en el Colegio del Rosario, es decir, antes de 1762.

No obstante, existe otro documento que hasta ahora ha pasado desapercibido para los historiadores de la ciencia y, en particular, de la astronomía en nuestro país lo que implica que al menos existió un criollo estudioso de temas astronómicos que no temió realizar modificaciones al modelo del universo imperante, construir las primeras efemérides astronómicas (fases lunares y eclipses) con tiempos referidos a una ciudad neogranadina y proponer una rectificación al calendario. El propósito de esta comunicación es exponer, con la debida perspectiva y análisis, el contenido de ese manuscrito.

Una aclaración con relación a la numeración para facilitar la localización de la información al interior del manuscrito. Este en la actualidad posee dos numeraciones en la parte superior derecha de cada folio que no son coincidentes: la de más arriba está escrita en tinta y es evidente que fue la que le dio el mismo autor; sin embargo, cada uno de los números está ligeramente tachado con lápiz, suponemos que por el mismo responsable de la segunda numeración, realizada en un tiempo posterior algo difícil de fijar pero, a juzgar por el folio que marcó con el número 1, con propósitos de catalogación; esta segunda numeración, realizada con lápiz, está ubicada inmediatamente abajo de la anterior (salvo en los últimos folios del manuscrito). El autor comenzó a enumerar los folios desde el inicio del tratado primero, por lo que los prólogos no están enumerados, a diferencia de la numeración a lápiz que sí los incluye y de ahí la diferencia. Aquí adoptaremos la numeración hecha en lápiz, pues permite hacer alusión a los prólogos; además, puesto que la numeración se realizó sobre la cara frontal de cada folio, esto es, del recto, y dado que el autor escribió en ambas caras del folio, para referirnos a la parte trasera correspondiente del folio (verso), marcaremos simplemente el número del folio seguido con la letra $\mathrm{v}$.

También aclaramos que las frases textuales del manuscrito se escribirán con la ortografía moderna para facilidad en la lectura.

\section{El manuscrito}

En la actualidad el documento reposa en la Biblioteca Nacional de Colombia; una versión digital completa de él puede conseguirse en la página web de la misma (http://catalogoenlinea.bibliotecanacional.gov.co/client/es_ ES/search/asset/107409/0, consultado el 9 de abril de 2019). Está redactado en castellano y aunque algunos folios evidencian el paso del tiempo, en su mayoría están en buen estado de conservación. Tiene unas dimensiones de $20.5 \times 13.5 \mathrm{~cm}$ y en su estado actual está constituido por 122 folios. Infortunadamente, a lo largo de los años fue perdiendo parte de su contenido. Eso se puede evidenciar por la ausencia de varios folios tanto al interior como al final del mismo.

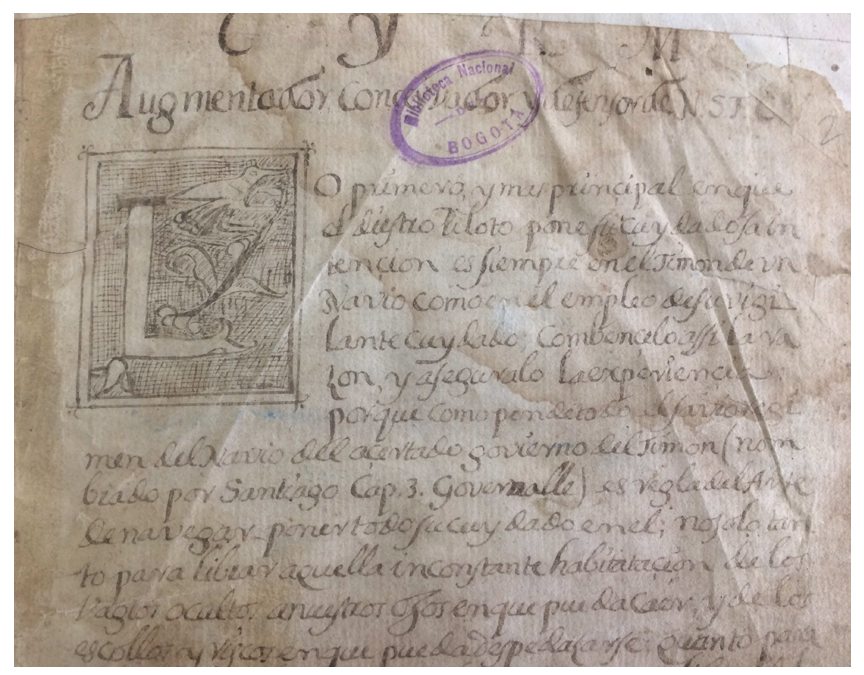

Figura 1: Una parte del inicio de manuscrito, el cual corresponde al prólogo dirigido al Rey

Podemos segmentar el contenido del manuscrito en las siguientes cinco partes.

1. Inicio dirigido al Rey y que lleva por título "CYRM [Católica y Real Majestad] Aumentador, conservador y defensor de NSFC [Nuestra Santa Fe Católica]”, (pág. 2 a 6v), (fig. 1).

2. "Carta y prólogo al sensor", (pág. 7 a 9v).

3. "Prólogo al lector", (pág. 10 a 12v).

4. "Tratado primero de astronomía y de la reformación del tiempo. Primera parte". Está conformado por trece capítulos; (pág. 13 a 44v). Están ausentes tres folios, por lo que el segundo y el cuarto capítulos están incompletos y el tercero está ausente en su totalidad.

5. "Tratado segundo de astronomía y de la reformación del tiempo. Segunda parte". Está conformado por diecisiete capítulos; (pág. 45 a 77v).

6. "Tratado tercero de astronomía y de la reformación del tiempo. Tercera parte". Está conformado por ocho 
capítulos; (pág. 78 a 123v). El octavo capítulo está incompleto.

Sabemos que en el tratado tercero había al menos un capítulo adicional (un noveno, ver pág. 15 y 57), hoy en día perdido, por lo que no se descarta que en su redacción original existieran otros capítulos adicionales e incluso al menos una cuarta parte. Sin embargo, esto último es poco probable, pues en las tres partes se cubrió la totalidad de los temas mencionados en los prólogos.

Por un comentario existente en la pág. 7 y otro más en la pág. 66 se puede colegir que el manuscrito se constituye en una quinta versión extendida de cuatro anteriores que previamente había remitido "por diversas manos" al rey español. Se ignora el paradero de esas versiones y si llegaron en algún punto a alcanzar las manos de su destinatario; una indagación elevada al Archivo General de Indias de Sevilla permitió verificar la ausencia de documentación al respecto.

El manuscrito es de difícil y ardua lectura. La obvia existencia de arcaísmos, un manejo algo arbitrario de los signos de puntuación y la letra del autor obstaculizan para el lector moderno una lectura ágil del escrito que permita de forma eficiente asimilar su contenido. Aunado a lo anterior está el hecho de que el autor carecía del don de la claridad y de la estructuración, de modo que el lector se expone, a lo largo del contenido, a no pocas repeticiones, ideas fuera de contexto y descripciones difíciles de digerir o de entender.

No es claro cómo llegó a sobrevivir el documento en los primeros 150 años después de su redacción. En la última línea de la página 33v se encuentran las palabras "Libro De Leonidas Cardenas" (sic) en una caligrafía distinta a la del autor, por lo que es de suponer que esta persona fue su dueño en algún momento del siglo XVIII o XIX. Posteriormente, a mediados del siglo XIX, y a través de un regalo del Señor Elías Prieto, quien vivía en la población de Soatá (Boyacá), llegó a las manos del literato José María Vergara y Vergara quien no dudó en incluirlo en su libro dedicado a la historia de la literatura en Nueva Granada (Vergara \& Vergara, 1867). Vergara \& Vergara enfatizó la "erudición del autor en conocimientos astronómicos" y la intención de este en corregir el cómputo cronológico y ofrecer una nueva fecha de la edad del mundo. Agregó que el libro "tiene tesis muy altas hasta cuestiones muy ridículas" pero reconoció que expuso "verdades revolucionarias en aquel tiempo". También mencionó que el manuscrito contiene tablas de eclipses, fases lunares y cómputos eclesiásticos y una tabla de la situación de algunas poblaciones del Nuevo Reino, aunque erróneamente señaló que estas estaban referidas a Madrid. También incluyó un pequeño extracto - con una extensión de unas treinta líneas- del prólogo dirigido al Rey.

Pocas décadas después, en su cuaderno de apuntes No. 13, el cubano José Marti hizo una alusión al libro de Sánchez de Cozar, ignoramos si con base en la información que halló en la obra de Vergara \& Vergara o de otra fuente. Marti, después de resumir escuetamente las aportaciones del libro, anotó: "Su estilo no es en ninguna manera literario; - sino revuelto, y cargado de indisculpables repeticiones, y muy pobre" (Marti, 1991).

Pinzón (1993) reprodujo las tres primeras partes en que hemos dividido el manuscrito (i. e., los prólogos). Agregó que estaba en curso un proyecto para publicar el resto del documento una vez terminado su estudio pero, al parecer, y si es que tal tarea fue llevada a cabo, esta nunca salió a la luz. Un análisis general y preliminar del manuscrito fue publicado recientemente (Moreno, Portilla, Zitzmann, 2018) en el que se dieron a conocer varios aspectos de su contenido.

\section{El autor}

El autor del manuscrito fue un sacerdote de nombre Antonio Sánchez de Cozar Gómez, aunque, en el prólogo al sensor, en la parte final, colocó en lugar de Gómez el apellido Guanienta con el propósito de mostrarse como descendiente directo del cacique indígena de ese nombre (Figura 2). Lo muy poco que sabemos sobre su vida quedó consignado por él mismo en lo que redactó al inicio del manuscrito. Dice haber nacido en el sitio de Mochuelo "en que se ha fundado por los míos y otros la nueva Villa de Santa Cruz de San Gil" (pág. 4), hoy San Gil (Santander); sin embargo, su sitio de residencia, al momento de redactar el manuscrito, era la población de Vélez. También afirmó poseer el cargo de "comisario particular subdelegado de la santa cruzada" (pág. 6), título que ostentaban numerosos curas y vicarios de poblaciones bajo el dominio español. Su padre fue Martín Sánchez de Cozar, nacido en la población española de Villa Nueva de los Infantes; su madre, de nombre Isabel Gómez Pabón, era descendiente directa del poderoso cacique Guanienta y aun vivía al momento de la redacción final del manuscrito; menciona que tenía hermanos, uno de los cuales se llamaba Martín (pág. 5v).

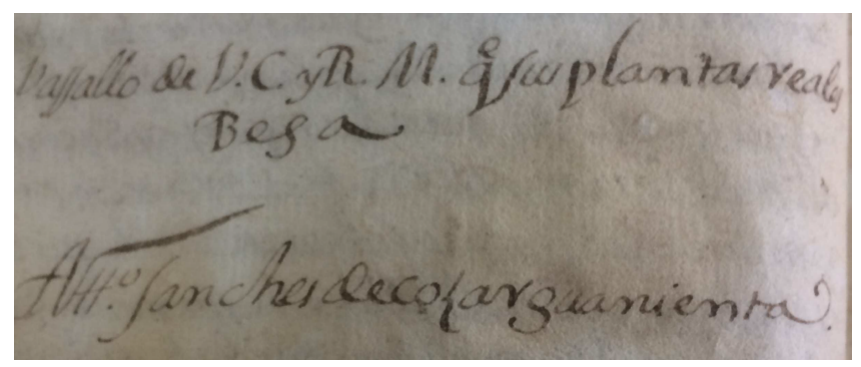

Figura 2: Parte final del prólogo dirigido al Rey en el que se consigna el nombre de su autor

Aparte de la anterior información, desconocemos prácticamente todo sobre su vida, incluidos el intervalo de tiempo en el que vivió, la orden a la que pertenecía y la formación académica que recibió, si es que la tuvo. Muy posiblemente dominaba a cabalidad el latín si hemos de basarnos en varias frases que redactó en ese idioma - y que 
las diferenció subrayándolas- a lo largo del manuscrito. Dejando a un lado sus conocimientos básicos astronómicos y de cronología, los que ciertamente no eran desdeñables, es claro también que dominaba la aritmética con cierta fluidez, la que le fue útil, entre otras cosas, para confeccionar las tablas de futuras fases lunares y eclipses, aunque ello no impidió que incurriera en errores de cálculo con cierta frecuencia. Tal y como ya se mencionó, Sánchez de Cozar no registró aspectos que aludan a que alguna vez recibió algún grado de formación normativa que, de haber sido relevante, muy seguramente hubiera anotado, por lo que suponemos que la mayor parte de sus conocimientos fueron adquiridos de forma autodidacta.

También se evidencia un afán de proyectar para sí una imagen de importancia habida cuenta de ser descendiente directo de un aguerrido cacique indígena (pág. $5 \mathrm{v}$ ), lo que no parece entrar en contradicción con la manifiesta condición de pobreza en la que vivían él y su familia (pág. 6).

Una pesquisa realizada por uno de los autores (Moreno) en las parroquias de las poblaciones de Vélez y de El Socorro, con el propósito de recoger algún indicio adicional sobre su vida, no arrojó ningún resultado. Es dudoso, entonces, que haya sido párroco de la iglesia de Vélez y en su lugar lo haya sido de una parroquia cercana a esta población o de San Gil. En al menos dos partes (pág. 6 y 7) Sánchez de Cozar dejó consignado que invirtió veinte años en la redacción completa del manuscrito, cuya finalización probablemente ocurrió en el año de 1696, ya que al final de la "Carta y el prólogo al sensor" colocó por fecha el 25 de diciembre de ese año (pág. 9v). Del mismo modo, en las páginas $71 v$ y 72 manifiesta que lo que está redactando lo hace en ese año.

Es evidente que Sánchez de Cozar debió tener acceso a un número importante de libros si hemos de juzgar por el abanico de autores que menciona a lo largo del manuscrito. De lo que se colige que debió tener la facilidad de consultar una nutrida biblioteca, probablemente de naturaleza monacal. Es incuestionable que el libro "Cronología y reportorio de la razón de los tiempos" de Rodrigo Zamorano (Zamorano, 1594) cuya primera edición data de 1594, influyó decididamente en Sánchez de Cozar, si tenemos en cuenta no solo las numerosas líneas que adaptó de ese libro sino también la imitación de su estructura y la forma de presentación de ciertas tablas.

De acuerdo con lo que anotó en uno de los prólogos, su intención en un comienzo era escribir solo para sí (pág. 3). Pero luego fue animado por otras personas a que comunicara sus ideas y visibilizara sus escritos. Al principio estuvo renuente a ello pues se sentía intimidado frente al amplio número de investigadores que hubo antes que él trabajando en esos temas. Pero después reconsideró su postura, le dedicó el libro al Rey y le solicitó a éste que corriera con los gastos de la impresión y, debido a la importancia que su autor valoraba sobre su contenido, un ejemplar debía ser mandado a Roma para ser examinado por el Papa. Todo parece indicar que ese anhelo nunca fue satisfecho.
Como ya se dijo, al sacerdote le tomó cerca de dos décadas redactar su obra, lo cual no es de extrañar si se tiene en cuenta que escribió (al menos hasta 1696) cinco versiones de la misma, junto con la elaboración de efemérides astronómicas, consistentes, como veremos, en fases lunares y eclipses por un número sustancial de años, así como la redacción de tablas perpetuas para calcular el número áureo - este concepto se examinará más adelante y tiene que ver con el ciclo de Metón y no debe confundirse con la proporción áurea - y las epactas (la edad de la Luna contada en días, el primero de enero, a partir de la última Luna nueva) dentro de la reforma al calendario que él propone.

Antes de entrar a la descripción formal de los capítulos que conforman cada uno de los tres tratados, es preciso dejar en claro que si bien la redacción del manuscrito ocurrió a finales del siglo XVII, esto es, para un tiempo en el que Copérnico, Kepler y Galileo ya habían fenecido y en el que los escritos físico matemáticos de Newton llevaban poco tiempo de ser redactados y publicados, la astronomía de Sánchez de Cozar es enteramente ptolemaica e inmersa aún en un fuerte escolasticismo, aunque, como examinaremos más adelante, pincelada con toques de originalidad. Desconocemos si el autor estaba al tanto de la existencia de la teoría heliocéntrica aunque, si ese hubiese sido el caso, lo más probable es que la hubiera descartado de entrada dado su firme convencimiento de no aceptar todo aquello que fuera en contra de las sagradas escrituras.

La relevancia de nuestro autor, como se verá más adelante, no reside en haber propuesto o defendido un nuevo modelo cosmológico. Consiste más bien en que él ofreció, libremente y sin ir en contra del canon establecido, varias ideas que le parecieron más plausibles acerca del movimiento y conformación de lo que se consideraba en ese entonces como universo. Su libertad residió en que sus amplias lecturas le mostraron que no fueron pocos los autores a lo largo de las centurias que se atrevieron a especular sobre aspectos que la Biblia y otros libros de doctores de la iglesia no dejaron en claro. Sánchez de Cozar juzgó que, a pesar de ser un aislado habitante de una oscura colonia española, nada en principio le prohibía ofrecer hipótesis plausibles sobre el conocimiento del cosmos imperante sin entrar en contradicción con los dogmas fundamentales de la iglesia. E incluso, como veremos, estaba convencido que el calendario gregoriano adolecía de varias deficiencias y que él, con base en una atenta lectura de las sagradas escrituras, podía ofrecer unas reglas alternativas que solucionaban para siempre el problema de la determinación de la Pascua de resurrección que celebran los católicos.

Lo que viene a continuación es una descripción resumida de cada tratado, lo cual permite hacernos una idea de los distintos temas que Sánchez de Cozar abordó y, al mismo tiempo constituya en una guía conveniente al interesado para facilitar la localización de la información. 


\section{El "Tratado primero"}

El propósito esencial del primer tratado es describir varios conceptos fundamentales de la astronomía de posición aunado a una descripción cosmológica y dinámica de los objetos que lo integran. Sin embargo, no está exento de exponer algunos aspectos de naturaleza astrológica. Es de particular interés la forma como el autor atribuye el movimiento a las esferas que contienen los planetas, junto con su propuesta de incluir un nuevo cielo entre la Luna y Mercurio. En tanto que no entren en contradicción con el texto bíblico y la opinión de varios doctores de la iglesia, el autor no teme consignar su opinión con relación a la forma y el tamaño de la Tierra, así como la ubicación a la que están situadas las estrellas.

En el primer capítulo expone varios aspectos básicos astronómicos comenzando con una descripción general de lo que es el universo. La visión es geocéntrica con claros rasgos aristotélicos. Los cielos "son unos cascos huecos de materia cristalina incorruptible, de figura redonda esferal discontinuos unos de otros, y continuos en sus partes [...] unos entre otros desde el Empíreo hasta el de la Luna en cuyo centro están los 4 elementos". Y a continuación esboza una característica que le otorga a tales cielos, pues cada uno (salvo el Empíreo) "tiene un ñudo en forma piramidal hacia la Tierra", el cual es responsable de que cada uno de esos cielos haya perdido estabilidad y esté dotado del movimiento que los caracteriza de oriente a occidente (pág. 13). Tanto el Empíreo (el más externo de los cielos, lugar de residencia del Dios cristiano, sus ángeles y los bienaventurados) como la Tierra son fijos e inamovibles. Define a continuación el punto céntrico de la gravedad al que otorga una gran importancia, pues a él le atribuye la forma circular de los cielos, así como su movimiento (pág. 13v).

A lo largo de este primer capítulo aparecen las definiciones de zodiaco, constelación, signo zodiacal, planeta (el cual define como un cuerpo redondo, hueco, lleno de una materia umbrosa y encajado en cada pirámide de su cielo correspondiente (pág. 15)), equinoccios, solsticios (estos dos definidos como círculos), declinación (que define como el movimiento oblicuo que hacen del ecuador a los trópicos cuya distancia es de $23,5^{\circ}$ ), spira, polos, cenit, almicantarat, la igualdad entre la altura de los polos con la latitud, paralelos, coluros mayores y menores y la eclíptica. No es raro que en las primeras definiciones existan comentarios sobre las "influencias buenas y malas" de los planetas y las estrellas. También introduce el "cielo incógnito" el que ubica entre Mercurio y la Luna (pág. 15 y 15v); adicionalmente, explica el funcionamiento de los eclipses tanto de Luna como de Sol (lo que acompaña con varias "demostraciones" (figuras)) y define la Vía Láctea denominándola "línea láctea" y aventura una explicación sobre su extraña coloración. Remata el capítulo definiendo los conceptos de auge y opuesto de auge y del crepúsculo matutino y el vespertino.

En el segundo capítulo describe la división del mundo en tres partes: invisible (el Empíreo), celeste (desde el cielo de las estrellas fijas hasta la Luna) y elementar (la Tierra). Menciona que el mundo está compuesto de 14 cuerpos circulares de los cuales nueve son los cielos "agentes" (el cielo estrellado, Saturno, Júpiter, Marte, Sol, Venus, Mercurio, el incógnito y la Luna) y los cuatro cuerpos restantes los identifica con los cuatro elementos aristotélicos. Por la ausencia de varias folios, ignoramos el contenido de lo que resta de ese capítulo, al igual que el contenido total del tercero. Del cuarto capítulo conocemos el contenido de menos de una hoja en el que se observa su insistencia en que, para su movimiento, los cielos no requieren de "inteligencias angélicas". En una línea escribe la palabra "epiciclo" pero en un contexto algo confuso.

En el capítulo 5 Sánchez de Cozar explica con más detalle la forma geométrica que deben tener cada uno de los cielos. Como se explicará más adelante en la sección de análisis, para el autor tanto la "grosesa" (grosor) que ha de tener cada cielo como el peso de cada planeta y el ñudo que lo envuelve son fundamentales para efectos de que hayan perdido la estabilidad y cada uno posea una velocidad distinta de su movimiento. También introduce un auto reparo, en el que explica el porqué de su atrevimiento de sugerir que los cielos se mueven sin necesidad de requerir "inteligencias angélicas".

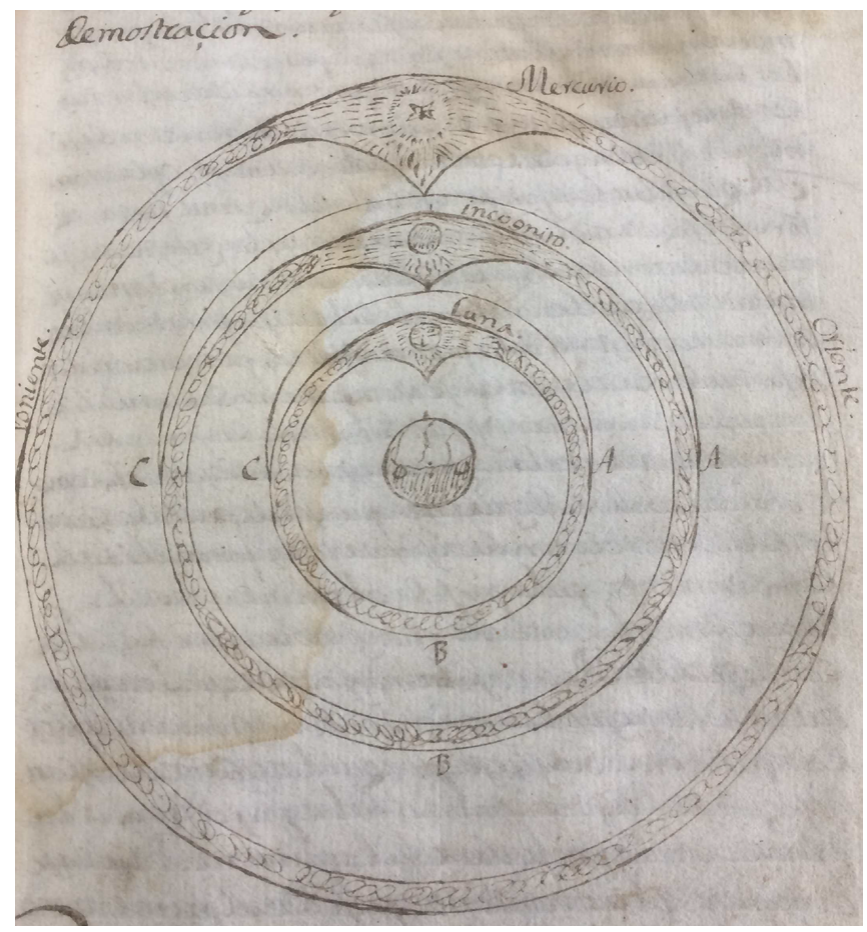

Figura 3: Imagen de la pág. 30 en la que muestra el cielo de la Luna, el cielo incógnito y el cielo del planeta Mercurio interactuando mutuamente a través de sus puntas piramidales

A lo largo del capítulo 6 ofrece más detalles sobre la concepción dinámica del movimiento de los cielos. Aquí exhibe 
una gran intuición física y el uso de un experimento mental para defender sus explicaciones acerca de los cielos dotados de movimiento circular alrededor del punto céntrico de la gravedad que está ubicado en el interior de la Tierra. Reitera su introducción de un cielo no contemplado antes, ubicado entre la Luna y Mercurio y que, como ya comentamos, denominó "el incógnito", motivado por la aparición de dos cometas que surcaron los cielos en 1681 y 1682 . Expone tres preguntas que los conocedores de sus ideas le habrán hecho e incluye sus correspondientes respuestas. Al responder esas preguntas ofrece más detalles de su concepción mecánica del movimiento de los cielos (Figura 2), aunque no está exento de introducir elementos muy simplistas para explicar la propensión de los cuerpos planetas a mantenerse dentro de la cinta del zodiaco.

El capítulo 7 está reservado para explicar el procedimiento por el cual, en el fin de los tiempos, caerán las estrellas del cielo y los cielos que contienen los planetas dejarán de moverse, en consonancia con lo descrito en el evangelio de Mateo $(24,29)$. De manera que el autor, de conformidad con su explicación dinámica del movimiento de los cielos, vislumbra un escenario en el que el ñudo de las estrellas fijas caerá sobre el ñudo de Saturno, de tal suerte que todo el sistema tendrá una estabilidad perfecta con respecto al punto céntrico de la gravedad y, por lo tanto, a partir de ahí todo el sistema estará estático por siempre.

Sánchez de Cozar dedica el capítulo 8 a describir el tiempo que le toma al cielo estrellado dar una revolución completa con respecto al Empíreo, al igual que los tiempos que les toman a los planetas hacer una revolución completa con relación al cielo estrellado. Con base en tales tiempos calcula la tasa angular diaria de cada uno de ellos. Para el primer caso asume que al punto vernal, que estaba en el cenit del campo damaceno (i.e., lugar que la Biblia fija como aquel en el que Dios creó a Adán) en el momento de la creación del mundo, le toma cerca de 7.000 años volver a pasar por el mismo sitio con respecto al Empíreo; lamentablemente, no explica la razón de asumir que dicho periodo es exactamente de 2.555 .000 días, $15^{m}$ y $6^{s}$. Luego calcula la tasa diaria de ese desplazamiento, que el autor se toma el trabajo en calcular en: $0^{\circ} 0^{\prime} 0^{\prime \prime} 30^{\prime \prime \prime} 26^{i v} 3^{v} 59^{v i} 31^{v i i} 49^{v i i i} 11^{i x} 52^{x}$. Como aclaración: la nomenclatura que usamos aquí para las medidas angulares es la siguiente: ' grado, ' minuto, " segundo, ${ }^{\prime \prime \prime}$ tercero, ${ }^{i v}$ cuarto, ${ }^{v}$ quinto, ${ }^{v i}$ sexto, ${ }^{v i i}$ séptimo, ${ }^{v i i}$ octavo, ${ }^{i x}$ noveno $\mathrm{y}^{x}$ décimo. Segundo, como se sabe, es la sesentava parte del minuto; luego tercero es la sesentava parte del segundo; cuarto es la sesenta ava parte de tercero y así sucesivamente. Con relación a las medidas de tiempo, tenemos que la notación es: ${ }^{d}$ día, ${ }^{h}$ hora, ${ }^{m}$ minuto, ${ }^{s}$ segundo, ${ }^{t}$ tercero, ${ }^{c}$ cuarto, ${ }^{q}$ quinto, ${ }^{s x}$ sexto, ${ }^{s p}$ séptimo, ${ }^{o c}$ octavo, ${ }^{\text {no }}$ noveno y de décimo.

Así mismo, el autor toma para los planetas los siguientes tiempos de revolución junto con sus correspondientes tasas diurnas de movimiento, que aquí escribimos con una notación más conveniente pero con los valores que el autor es- cribió y calculó: Saturno $\left(10767^{d} 8^{m} 32^{s}, 0^{\circ} 2^{\prime} 0^{\prime \prime} 22^{\prime \prime \prime} 4^{\prime v} 2^{v}\right)$, Júpiter $\left(4330^{d} 11^{m} 48^{s}, 0^{\circ} 4^{\prime} 59^{\prime \prime} 18^{\prime \prime \prime} 25^{\prime v} 46^{v}\right)$, Marte $\left(686^{d}\right.$ $\left.22^{m} 57^{s}, 0^{\circ} 31^{\prime} 29^{\prime \prime} 12^{\prime \prime \prime}\right)$, Sol, Venus y Mercurio - nótese que toma los tres objetos con los mimos valores- $\left(365^{d} 5^{h} 50^{m}\right.$, $\left.0^{\circ} 59^{\prime} 8^{\prime \prime} 14^{\prime \prime \prime}\right)$, incógnito $\left(300^{d}, 1^{\circ} 12^{\prime}\right)$ y la Luna $\left(27^{d} 7^{h} 43^{m}\right.$ $\left.48^{t}, 13^{\circ} 10^{\prime} 56^{\prime \prime}\right)$. Adviértase que, curiosamente, en varios casos, al dar los periodos, el autor omitió los valores correspondientes a las horas. También incluyó el ángulo que cada planeta describe en el periodo con un número entero de días y la tasa horaria de desplazamiento que no hemos aquí reproducido. Al parecer, para la determinación de estos valores el autor tomó como modelo los datos contenidos en la tabla de la pág. 56 del libro de Juan de Pineda (De Pineda, 1589) $\mathrm{y}$, por las sutiles diferencias que existen al comparar los valores, se puede inferir que Sánchez de Cozar no se conformó con simplemente copiarlos de ese libro.

Datos sobre el número de estrellas, así como su división en términos de su brillo (y con esto, de su tamaño), al igual que el tamaño de los planetas con relación al diámetro de la Tierra están contenidos en varias tablas del capítulo 9 . Además, en forma también de tabla, incluye las distancias de los planetas a la Tierra, así como la "grosesa de los cantos" de cada uno de ellos, Manifiesta que los valores que presenta son tomados tanto de Ptolomeo como de Alfagano (Al-Farghani) e incluye también los datos de distancia los planetas de la obra de Juan de Pineda.

El capítulo 10 es dedicado a un análisis sobre el brillo y las distancias a las que han de estar situadas las estrellas. Sánchez de Cozar cuestiona los valores de distancias que han ofrecido los autores que ha consultado basándose en suponer cómo sería el brillo del Sol si estuviese a tales distancias, llegando a la conclusión de que este no se llegaría a observar. De modo que tiene dos opciones: o las estrellas son "mucho mayores que el Sol" o, en su defecto, están más cerca de lo que se ha asumido. El autor desecha la primera hipótesis ("siendo como son algo menores") y aunque adopta los valores de las distancias de los cuatro primeros objetos más cercanos a la Tierra (la Luna hasta el Sol) que ofrece Juan de Pineda, reduce significativamente los valores de las distancias a los planetas Júpiter y Saturno y, de forma notoria, reduce a una sexta parte la distancia a la que está situada la superficie "convexa" del cielo estrellado.

La discusión sobre la forma y el tamaño de la Tierra la reserva para el capítulo 11 . Frente a los distintos valores que diferentes autores han propuesto para la longitud de la circunferencia de la Tierra $(6.300,7.500,8.912$, en unidades de leguas) Sánchez de Cozar llega a una conclusión sorprendente: todos esos autores están en lo correcto. Y la razón es que la Tierra, estrictamente hablando, no es una circunferencia perfecta: hay unas regiones que están significativamente más elevadas que otras. El autor, con base en una descripción que un viajero hizo en las tierras del "Perú y Lima" en donde apreció que "avanza por allí el Sol en una hora 370 leguas y más un tercio de otra por la razón de ser como son esas tierras las más elevadas hacia la región media 
que las demás", infiere, con razonamientos algo confusos, que la longitud de la circunferencia en tal lugar de la Tierra es de $370.33 \times 24 \approx 8888$ para lo cual le da un radio terrestre en esa zona igual a $8.888 / 6,283 \approx 1.414$ leguas. No queda de más señalar que hay una amplia incertidumbre en establecer una equivalencia de esta longitud en unidades de longitud modernas, ya que la legua no solo variaba de forma notable entre los diferentes reinos españoles, sino que recibió a lo largo del tiempo distintas redefiniciones. Suponiendo que Sánchez de Cozar se refiriera a la legua castellana, que equivalía a unos $4,19 \mathrm{~km}$, tenemos que el radio terrestre para él era de unos $5.925 \mathrm{~km}$.

Los dos capítulos restantes, el 12 y el 13, son de muy escaso interés astronómico. El capítulo 12 está dedicado a exponer relaciones de carácter eminentemente astrológico; es así como expone varias configuraciones planetarias (conjunción, sextil, cuadrado, trino, oposito), pero tales conceptos no solo operan entre planetas sino algunos de ellos se dan entre las mismas constelaciones zodiacales reforzando o anulando las "cualidades" que poseen e influeyendo "las cosas de acá abajo". En el mismo sentido netamente astrológico explica los conceptos de "casas" y "haces" de los planetas. Agrega: "en alguna manera nos vienen los astros y planetas con que al tiempo de nacer nos cogen estas posiciones ascendiendo ellos por el oriente que en cuando influyen sus virtudes" (pág. 44). El capítulo 13 trata sobre los años "climatéricos" y tiene que ver con aspectos de naturaleza numerológica ya que, según tradiciones antiguas, existían ciertos años particulares a lo largo de la vida de un ser humano que eran determinantes o para la vida o para la muerte. El número 63, por ejemplo, se consideraba particularmente aciago para un hombre que cumpliera tal edad.

\section{El "Tratado segundo"}

El tema central del tratado segundo es el tiempo. Inicia el capítulo 1 con una definición del concepto de eternidad citando a san Agustín. Sin mayores preámbulos pasa al capítulo 2 donde define el evo como la duración de las cosas eviternas (aquellas que tienen un principio pero no un final, como los espíritus, ángeles, cielo Empíreo) y lo diferencia del concepto "tiempo", el que tendrá fin al momento en que los cielos dejen de poseer movimiento.

En el capítulo 3 especifica que el tiempo es la duración del evo y lo liga al concepto del movimiento de los cielos. Menciona que las "divisiones naturales" del tiempo son solo tres y corresponden al movimiento de los "dos luminares magnos", esto es, el Sol y la Luna. Estas tres divisiones son: 1) el día solar, que él define como el tiempo que tarda el Sol en dar una vuelta de oriente a ocidente partiendo de cualquier cenit hasta volver otra vez al mismo. Su duración es de $24^{h} 57^{s} 32^{t} 3^{c} 17^{q} 15^{s x} 37^{s p}$; 2) el año, entendido como el tiempo en que tarda el Sol en dos pasos consecutivos por el punto vernal, aunque afirma que es un movimiento de "oriente en poniente". A su vez este movimiento lo hace de cuatro maneras: i) el año natural propiamente dicho (con duración de $365^{d} 5^{h} 50^{m}$ ); ii) el ciclo de 19 años, que es claramente el ciclo de Metón (aunque no lo menciona con este nombre); iii) un ciclo de 62 años, y iv) un ciclo de 144 años que denomina "circulación magna del Sol", que es significativo para el autor puesto en él descansa parte de su propuesta de reforma del calendario. Como acabamos de ver, el autor adopta como valor de la duración verdadera del año, o más estrictamente del año trópico a $365^{d} 5^{h} 50^{m}$, que es con el que realiza, como veremos, su modificación al calendario. A lo largo del manuscrito lo escribirá siempre de ese modo, sin especificar los segundos, pero es claro que tomó $365^{d} 5^{h} 50^{m} 0^{s}$. Finalmente, el 3) corresponde al mes, y lo divide en dos: i) el mes sideral, con duración de $27^{d} 7^{h} 43^{m} 5^{s} 48^{t}$ y el mes sinódico, con una duración de $29^{d} 12^{h} 42^{m} 3^{s} 12^{t}$, aunque no utiliza propiamente esos nombres.

Una explicación más detallada del "día natural" está contenida en el capítulo 4. Repite su duración tal y como lo anotó en el párrafo precedente $\left(24^{h} 57^{s} 32^{t} 3^{c} 17^{q} 15^{s x} 37^{s p}\right)$. Tal parece que lo que Sánchez de Cozar quiso anotar aquí es la duración del día solar con respecto al día sideral (que es de unas $\sim 24^{h} 3^{m} 57^{s}$ ). La omisión de los minutos (o de las horas), curiosamente, es algo que no es raro en el autor, tal y como se anotó en el capítulo 8 del primer tratado. También define aquí el día natural como el tiempo que tarda el Sol "partiéndose de un cualquier punto del zodiaco y volviendo otra vez al mismo", es decir, parece confundirlo con la definición de año. Más adelante comenta que hay dos maneras de medir el inicio del día: o bien desde el mediodía ("el más perfecto modo de medir todo lo que es tiempo") y "al contrario: esto es, de media noche a media noche". Luego define el día artificial como aquel tiempo en que "el Sol nos alumbra desde que nace hasta que se pone" y comenta la diferencia de la duración de este tiempo que hay en el ecuador (donde hay igualdad de duración entre el día y la noche) con la que surge si un observador se va desplazando hacia los polos, en cuyos puntos es mayor la diferencia entre el día artificial y la noche.

El capítulo 5 está reservado a explicar las dos divisiones del día natural. La primera es la natural planetaria (o temporal desigual). La razón del nombre radica en que asigna a cada planeta (desde Saturno hasta la Luna) un efecto para cada hora a lo largo del día. El autor tiene especial preocupación por especificar el momento de la creación del mundo, e incluso fijar el meridiano (utiliza la palabra cenit) del lugar en el que el Sol culminaba al momento de la creación de este astro, esto es, el "campo damaceno" o campo de Damasco que, como ya se dijo, es comúnmente considerado el lugar donde fue creado Adán y presumiblemente ubicado en algún punto del Levante mediterráneo. Esto, para contar desde ahí las horas en que cada planeta "reina" exactamente sobre cada hora del día; como son siete planetas hay algunos de ellos que alcanzan a reinar hasta cuatro veces a lo largo de un día. Habiendo fijado las reglas en que cada planeta predomina en 
el meridiano de referencia (esto es, en el campo damaceno) a partir de la primera hora de tiempo local indica qué debe hacerse para determinar las horas de las entradas de cada planeta correspondiente a cualquier otro observador ubicado en la superficie del planeta. De ahí que remita al lector al capítulo 7 del tercer tratado donde, en varias tablas, están las longitudes en unidades de tiempo (así como latitudes) de numerosas ciudades tanto de España como de sus colonias. Al final de este capítulo comenta la otra manera de dividir el día natural: en horas artificiales (equinocciales) que, de acuerdo con la explicación ofrecida, está basada en un Sol que se desplaza por el ecuador celeste, esto es, una especie de día solar medio y que hace que las horas posean igual duración.

Varias formas de dividir la duración del día, segmentado en horas equinocciales, se explican en el capítulo 6. La primera es semejante a como la usamos hoy: cada hora segmentada en 60 partes llamadas minutos; cada minuto segmentado en 60 partes llamados segundos, cada segundo segmentado en sesenta partes denominados "terceros", y así sucesivamente. De hecho, describe esta forma en dos ocasiones y en lugares distintos del capítulo, en la segunda ocasión llega en ese proceso de fragmentación a subdividir hasta los "décimos". La segunda forma consiste en dividir cada hora en cuatro cuartos ( 1 cuarto $=15$ minutos $)$; cada cuarto a su vez es dividido en diez partes llamadas momentos $(1$ momento $=1,5$ minutos $)$; cada momento es dividido en 12 partes denominadas uncías ( 1 uncía $=7,5$ segundos) y cada uncía es dividida en 44 partes llamadas átomos (1 átomo $=0,17045$ segundos). También menciona que en lugar de dividir la hora en cuatro cuartos, algunos la dividieron en cinco quintos ( 1 quinto $=12$ minutos). Esto último, al parecer, tiene que ver con el esfuerzo que hicieron algunos en relacionar el día artificial con el "día lunar", este último definido como el tiempo entre dos salidas consecutivas de la Luna por el horizonte oriental, que el autor estima más largo con relación al día artificial en $4 / 5$ de hora. En el capítulo 7 describe otra formas de fraccionar la duración del día consistente en dividirlo en cuatro segmentos de seis horas cada día, en consonancia con las cuatro épocas del año, las cuatro edades del hombre, etc. Complementa con una nueva propuesta de cómo pueden ser los intervalos de hora que han de tener esas cuatro fases y las relaciona con las edades del hombre (Figura 4).

El capítulo 8 contiene una discusión sobre las fechas exactas en que, según el autor, han de sucederse tanto los equinoccios como los solsticios. Sánchez de Cozar no comparte el que algunos cronólogos hayan fijado el equinoccio de primavera el 21 de marzo y el equinoccio de otoño el 23 de septiembre, lo que implica que el Sol se tarda más de tres días en ir hacia el trópico de Cáncer que hacia el trópico de Capricornio. Esto es inadmisible para el autor, pues, según él, "el movimiento del Sol es el más perfecto que el de los demás planetas" y no es concebible que el astro dador de luz se le "hubiese dado la imperfección de ser más tardo hacia el uno que hacia el otro". El autor establece el equinoccio en términos de la fecha de creación del mundo (que él fija, a lo largo del manuscrito, en un 20 de marzo) por lo que acomoda el equinoccio entre el 20 y el 21. Aunque, de acuerdo con el Génesis, Dios creó el Sol (y la Luna) solo hasta el tercer día, justifica su elección dando mayor peso a la luz que se creó desde el primer día. Además, comenta que tanto el solsticio de junio como el solsticio de diciembre están relacionados con la fecha de concepción y de nacimiento de Juan el Bautista. Establece que las fechas tanto de equinoccios como de solsticios son: 21 de marzo, 20 de septiembre, 21 de junio y 21 de diciembre y que de ahí no se deben salir. Discute también algunos aspectos de la modificación del calendario juliano y gregoriano. A manera de aclaración es conveniente señalar que, en realidad, los equinoccios y los solsticios no caen exactamente en los mismos días del año; así mismo, se verifica que, en promedio, el Sol sí invierte cerca de 90 días en ir del solsticio de diciembre hasta el equinoccio de marzo mientras que gasta 93 días en desplazarse entre el solsticio de junio al equinoccio de septiembre. Esto se debe a la velocidad variable de la Tierra alrededor del Sol, la que es mayor al inicio del año.

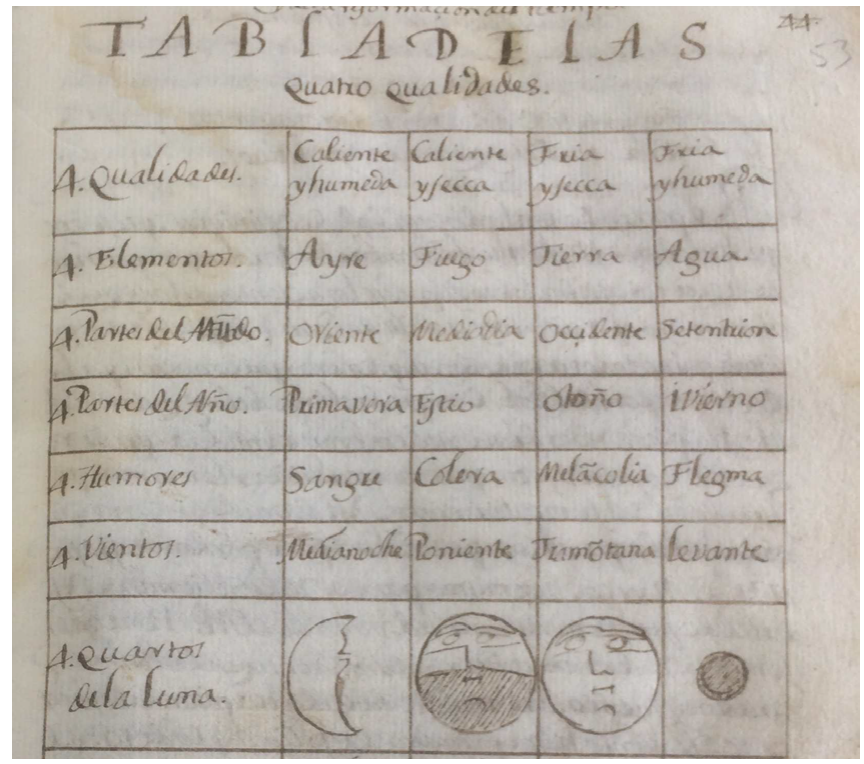

Figura 4: Tabla de las cuatro cualidades, según se explica en el capítulo 7 del segundo tratado, pág 53

Una amplia exposición de carácter numerológico se puede encontrar en el capítulo 9. Comienza por definir el concepto de semana como el "tiempo en que Dios creó al mundo"; procede a continuación a narrar lo que Dios fue creando del universo en términos de 6 días (con uno adicional de descanso), ajustado, como es de esperarse, a la narración del Génesis. El número 7 adquiere, por lo tanto, un importante significado, por lo que el autor narra diversos eventos bíblicos en los que está involucrado dicho número, aunque también cita algunos en los que aparece el número 14 (“de 
siete en siete") y el número 70. Esta exaltación del número siete constituye en un preámbulo para exponer las "tablas perpetuas" (ver pág. 59v), las cuales están conformadas por siete columnas con el propósito de encontrar, para un año determinado, la "letra dominical" (cualquiera de las siguientes siete letras: A, B, C, D, E, F, G, con una ligera modificación en los años bisiestos) y el "ciclo solar" (de ahí que la tabla esté constituida por 28 líneas). Estos dos conceptos sirven de instrumento en la cronología cristiana para la determinación de la fecha de la Pascua de resurrección.

Sin embargo, la tabla en referencia no es muy clara en su uso, y las explicaciones anexas de la misma no permiten ofrecer con claridad su uso correcto. Agravando el asunto está el hecho de que la misma contiene ya "su reformación", esto es, la modificación que él propone al calendario, la que solo explica con detalle varios capítulos después. Al final del capítulo hay una tabla (pág. 60v) en la que se exhiben el número áureo, la letra dominical y la epacta para varios años a intervalos de 144 con dos secuencias de conteo: a la izquierda el conteo es desde el año de la creación del mundo (año 1) hasta el año 3.744 después de esa creación; a la derecha, con los años contados en la era cristiana, también a intervalos de 144 años a partir del año 1.

Una explicación más detallada de la tabla de la pág. 60v se nos ofrece en el capítulo 10. El autor utiliza el concepto de epacta para años anteriores a 1582 de modo que calcula un valor de ésta igual a 11 para el año primero de la creación del mundo, lo que es consistente con la fecha de creación de la luz un 20 de marzo y de la creación de la Luna y el Sol tres días después; esto a la vez es consistente con una conjunción que ha debido ocurrir el día 18 de marzo (lo que está de acuerdo con una posición de la Luna unos $65^{\circ} 54^{\prime} 40^{\prime \prime}$ (pág. 55) al oriente del Sol en el momento en que Dios creó ambos astros). Un amplio espacio de este capítulo está dedicado a justificar por qué asignó determinados valores de epacta a ciertos años. Al final se consignan dos tablas en las que aparecen los valores de las epactas "desmesadas", una correspondiente para el año 1 hasta el 3744 (pág. 63v) y otra a partir del año $68 \mathrm{~d}$. C.

La discusión sobre las siete edades del mundo está contenida en el capítulo 11. El autor divide la historia en seis segmentos dejando el séptimo para el "descanso de la eternidad" después del fin del mundo. El devenir histórico está, por supuesto, enmarcado por los sucesos relatados en la Biblia, de modo que el inicio y el final de cada época está definido por eventos que el autor juzga decisivos, tales como el diluvio universal, la destrucción de Jerusalén y el nacimiento de Cristo. Pero, para efectos de fijar lo más exactamente posible los intervalos de cada edad, Sánchez de Cozar juzga imprescindible determinar el año exacto del nacimiento de Cristo, labor complicada habida cuenta de las varias dificultades que se interponen para alcanzar el éxito en la tarea. Entre ellas están la amplia diversidad de fechas que se han propuesto para tal evento, la pérdida de información fiable a causa de las convulsiones históricas y el uso reite- rado del mismo nombre para bautizar a distintos reyes a lo largo de la historia. Aquí nos enteramos de que el autor ha abordado el tema de la fecha del nacimiento de Cristo en cuatro anteriores cuadernos, pero reconoce que en ellos incurrió también en error como muchos otros computistas (ver pág. 66). Fija entonces como fecha de nacimiento de Cristo al año 3821 después de la creación del mundo y a continuación establece lo que a su juicio son los intervalos correctos de las edades del mundo. A lo largo de las siguientes cuatro páginas otorga, de acuerdo con lo anterior, las fechas en que ocurrieron, a partir de la creación del mundo, cerca de 30 distintos eventos narrados en la Biblia.

En el capítulo 12 explica la definición del número áureo $(N A)$ y su fundamento (la casi igualdad de 19 años solares trópicos con 235 meses sinódicos), y la manera como ha de calcularse para un año determinado, para lo cual utiliza una fórmula que aquí bien podemos escribir como:

$$
N A=\left(\frac{A+1}{19}\right)_{r},
$$

donde $A$ representa el año y el subíndice $r$ representa el resto ("sobra") de la división; en el caso en que con la fórmula anterior no se obtenga resto (i.e., en el caso en que $A+1$ sea exactamente divisible por 19) entonces el valor de $N A$ es igual a 19. Y muestra varios ejemplos de su aplicación. Insiste en su determinación de fijar el año del nacimiento de Cristo en el año 3821 después de la creación del mundo, en el que ha de corresponder a un número áureo igual a 2 y una exposición del porqué no es posible asignar otra fecha para ese evento (de las 10 que diversos autores habían propuesto antes que él).

Los conceptos de mes solar y de mes lunar son explicados en el capítulo 13. El mes solar que, como su nombre indica, está basado en el movimiento del Sol, lo divide en dos: i) el aparente y el ii) igual. El primero es el tiempo que gasta el Sol en atravesar los 30 grados que comprende cada signo del zodiaco; el segundo es aquel tiempo de igual duración en que haría lo mismo con una duración de $30^{d} 10^{h}$ $29^{m} 10^{s}$. El mes lunar sí está basado en el movimiento de la Luna; lo divide en tres: i) el peragratorio, que es lo que comúnmente se denomina mes sideral, con una duración de $27^{d} 7^{h} 43^{m} 2^{s} 48^{t}$ (en el capítulo 3 colocó $5^{s}$ en lugar de $2^{s}$ ); ii) el aparente, que es el tiempo transcurrido entre dos observaciones consecutivas de la Luna después del novilunio y que, por obvias razones, es desigual, y ofrece cuatro razones del porqué de esa desigualdad. Finalmente, explica el mes iii) consecutorio, que es el mes sinódico, con una duración de $29^{d} 12^{h} 44^{m} 3^{s} 12^{t}$ (en el capítulo 3 colocó $42^{m}$ en lugar de $44^{m}$ ) y describe los tiempos entre el cuarto menguante y el creciente, así como el tiempo transcurrido entre cada cuarto.

En el capítulo 14 se ocupa de examinar el concepto del año trópico $\mathrm{y}$, de forma extensiva, de su duración. Refiere distintas duraciones del año trópico, como las que ofrecieron Calipo, Aristarco y Arquímedes $\left(365^{d} 6^{h}\right)$, Hiparco y Ptolomeo $\left(365^{d} 5^{h} 55^{m} 12^{s}\right)$ y Albategni -i. e., 
Al-Battani- $\left(365^{d} 5^{h} 46^{m} 24^{s}\right)$. Al comentar sobre avances posteriores, en particular para el tiempo de la "reformación" gregoriana, menciona a Nicolás Copérnico, pero escribió Copernio. Este dato es significativo pues, al parecer, fue de la edición del libro de Zamorano de 1594 —o una reedición posterior- de la que Sánchez de Cozar extrajo esa información (pág. 103 de esa edición), pues la narración es parecida aunque no idéntica, y allí aparece escrita la palabra "Copernio"; es claro que se trata de un error de imprenta, ya que varias hojas más adelante aparece correctamente la palabra "Copernico" (sic). En la edición de 1621 se subsanó ese error. La citación de Copérnico, tanto para Zamorano como para Sánchez de Cozar, tiene que ver con el dato de la duración del año trópico y no con la teoría heliocéntrica.

Después de la alusión a Copérnico, Sánchez de Cozar menciona también las tablas pruténicas (tablas prusianas elaboradas por Erasmus Reinhold en 1551) a las que parece confundir con las tablas alfonsíes (que datan de mediados del siglo XIII), las que adoptaron un año con una duración de $365^{d} 5^{h} 49^{m} 16^{s}$. La narración no es clara en este punto, pero parece señalar que fue este el valor que adoptaron los astrónomos que asesoraron a Gregorio XIII para efectos de establecer la reforma gregoriana, porque a continuación comenta la estrategia para evitar que cada 400 años el año civil se adelante tres días con relación al trópico. Como se sabe, la reforma gregoriana estableció la eliminación de 10 días del mes de octubre de 1582 haciendo que del día 4 se pasara al 15 (para efectos de que el equinoccio cayera otra vez el 21 de marzo, pues se verificaba en esos tiempos el día 11 de ese mes); además, se fijó que aquellas centurias tales como 1700,1800 y 1900 , que no son exactamente divisibles por 400 , no fueran años bisiestos, de modo que con esa estrategia se busca que el año civil no exceda al año trópico por tres días, que era lo que sucedía con la utilización del calendario juliano.

Sin embargo, Sánchez de Cozar, un siglo después de la reforma gregoriana, considera que hay aspectos de las fases de la Luna que no concuerdan, por lo que llegó a la conclusión que la duración del año trópico ha de ser 44 segundos más larga (i.e., $365^{d} 5^{h} 50^{m} 0^{s}$ ), lo que probablemente acogió basado en un error de cálculo. Veamos: el autor procedió a determinar qué tanto se aparta el Sol del punto vernal al cabo de cuatro años. Así que realiza el cálculo de la tasa de desplazamiento diaria del Sol; obtiene $360^{\circ} / 365^{d} 5^{h} 50^{m} 0^{s}=0^{\circ} 59^{\prime} 8^{\prime \prime} 14^{\prime \prime \prime}$; luego, al cabo de 365 días civiles, el Sol habrá recorrido $359^{\circ} 45^{\prime} 37^{\prime \prime}$, pero Sánchez de Cozar comete un error, pues obtuvo $359^{\circ} 45^{\prime} 05^{\prime \prime}$ (i.e., 32" menos); por lo tanto, lo que se atrasa el Sol anualmente con respecto al punto vernal es $0^{\circ} 14^{\prime} 23^{\prime \prime}$ (Sánchez de Cozar obtiene $0^{\circ} 14^{\prime} 55^{\prime \prime}$ ); luego cada cuatro años el Sol se habrá atrasado con respecto al punto vernal $0^{\circ} 57^{\prime} 32^{\prime \prime}$; pero Sánchez de Cozar, con el error que lleva, obtiene $0^{\circ} 59^{\prime} 39^{\prime \prime}$, esto es, una diferencia significativa de $2^{\prime} 07^{\prime \prime}$. El sacerdote advierte que $0^{\circ} 59^{\prime} 39^{\prime \prime}$ no es igual a $0^{\circ} 59^{\prime} 8^{\prime \prime}$, por lo que, en poco más de una centuria ese acumulado hará que sea nece- saria la aplicación de una "simulación" del bisiesto, esto es, establecer que, aunque sea divisible exactamente por cuatro un año no se considerará bisiesto y volver con ello a hacer coincidir el Sol con el punto vernal. Sin embargo, aquí no hace el cálculo de cada cuánto hay que hacerlo (lo que hizo, como veremos, en el primer capítulo del tercer tratado, pero de otra forma); si lo hubiese hecho, hubiera advertido el error. Finalmente, el autor señala que el calendario gregoriano ha continuado perpetuando la costumbre de no celebrar el día de la Pascua en la fecha en que de verdad corresponde, y menciona que ello se debe a no haber "simulado" 11 días y 11 letras en el año de 1582, y agrega taxativamente "no basta decir que el año tiene tanta cantidad y que las simulaciones del bisiesto se deben hacer cada cierto tiempo sino se comprueba con traerlas sin discordancia alguna desde la creación del mundo hasta el tiempo presente".

La definición de año lunar está contenida en el capítulo 15. Lo diferencia en dos: el común y el embolismal. El común corresponde a la duración de 12 conjunciones de la Luna con el Sol, que equivale a $354^{d} 8^{h} 48^{m} 32^{s} 24^{t}$. Escuetamente Sánchez de Cozar anota que el embolismal corresponde a la duración de 13 conjunciones y que posee una duración de 384 días. Aunque el autor no lo menciona, ambos conceptos son de importancia en los calendarios lunisolares tales como el judío.

Lo relacionado con los periodos realizados "por las demás estrellas", es decir, por los planetas, el autor en el capítulo 16 menciona que tales periodos para cada planeta se definen de dos maneras: la primera es el tiempo que tardan en volver a pasar por el mismo punto de forma consecutiva por la esfera celeste; y la otra, de igual duración, es de clara connotación astrológica, pues está relacionada con el tiempo que dura el efecto que cada planeta en el que predomina y otorga sus influencias como causas segundas (la causa primera es Dios) sobre "los imperios, ciudades o cosas semejantes". Con relación a su duración refiere al capítulo 8 del primer tratado.

Finalmente, el capítulo 17 versa primeramente sobre la entrada de los cuatro tiempos del año, esto es, las estaciones. Comenta que tales tiempos de entrada de cada estación no son los mismos en las zonas templadas (en los que claramente se diferencian entre sí y tienen una continuidad de tres meses) que en regiones de baja latitud, en las que en la práctica no se dan con marcada diferencia y operan más las temporadas de lluvia y de sequía. Describe los intervalos de épocas de lluvia o de verano de la región que habita y los contrasta con los que se observan en España. Añade a esto una explicación de las celebraciones de tipo litúrgico que se efectúan a lo largo del año conocidas como las cuatro temporas: desviación, renovación, reconciliación y peregrinación. Su origen lo atribuye al papa Calisto para efectos de paliar la alteración de los "cuatro humores" de los seres humanos y por ello ayunar en cada una de ellas por espacio de tres días. 


\section{El "Tratado tercero"}

El tema del tratado tercero continua siendo el tiempo, pero con énfasis en la explicación de la reformación del autor, así como en la presentación de varias tablas de efemérides consistentes en los tiempos de las fases de la Luna (nueva y llena) y eclipses lunisolares a lo largo de un periodo que comprende 62 años. También llaman la atención las tablas de latitudes y longitudes, en particular las que corresponden a poblaciones del territorio del Nuevo Reino de Granada.

Ya visto con algo más de detalle, el capítulo 1 inicia con el recuento de la manera como la iglesia católica estableció la fecha de la Pascua, la cual ha de celebrarse el domingo siguiente a la luna llena después del equinoccio vernal (que se debe verificar el 21 de marzo). El autor escoge, como ya hemos visto, la duración del año trópico de $365^{d} 5^{h}$ $50^{m} 0^{s}$ y muestra que es posible establecer un acoplamiento con el calendario civil algo diferente al que se definió con la reforma gregoriana, de modo tal que cada 144 años sea necesario fijar una "simulación" del bisiesto (es decir, que pudiendo ser tal año divisible por cuatro y en cuyo caso conformado por 366 días, sea un año común de 365 días) para efectos de cuadrar de nuevo el Sol con el punto vernal. El razonamiento de Sánchez de Cozar es este: puesto que la duración del año es de $365^{d} 5^{h} 50^{m} 0^{s}=365,24305555$, cada cuatro años se acumulan $0,2430555 \times 4=0,972222$ días. Pero eso implica que se va adelantando el año civil del trópico en un valor de 1-0,972222 =0,02778, días, o sea, 40 minutos, cada cuatro años. Ello implica que al cabo de: $4 / 0,02778 \approx 144$ años se habrá acumulado un día.

En particular, propone que el año $68 \mathrm{~d}$. C. y a partir de ahí, con intervalos de 144 años, serán años normales de 365 días. De ese modo resultan años con esa misma característica, y antes del año de la corrección (1582), los años: 68, 212, 356, 500, 644, 788, 932, 1076, 1220, 1364, 1508 (ver parte derecha de la tabla de la pág. 60v). Ello hace que existan 11 de esos días. De ahí que el autor insista que en el año de la corrección no se debieron eliminar 10 días con sus letras; en lugar de ese número debieron haberse eliminado 11.

Más adelante el autor contrasta su propuesta con la del calendario gregoriano (y calcula para éste las sobras que se presentan a lo largo de cada cuatro años) y advierte que en ciertos años particulares sea difícil establecer la fecha de la Pascua, dado que el plenilunio de marzo puede ocurrir unas horas antes del 21 de marzo. En particular, muestra el caso para los años de 1704 y 1761. Sánchez de Cozar atribuye esta serie de desfases a la manera como se estableció el calendario gregoriano e insiste en que estas pueden ser superadas si se adopta la duración del año propuesta por él; el hacer "simulación" del bisiesto cada 144 años y fijar la fecha del nacimiento de Cristo en el año 3821 de la creación del mundo.

El propósito del capítulo 2 es explicar con más de detalle el concepto de las epactas, así como dar más argumentos en favor de su reformación del calendario. Inicia con una explicación de la diferencia de 11 días existente entre un año solar y doce meses sinódicos y el modo en que era posible conocer la edad de la Luna en el primer día del año con base en el número áureo. Aun cuando el uso exclusivo de este número conlleva a un error en el tiempo para determinar la edad de la Luna, expone la utilidad de ese concepto, bien para haber fijado, según él, el año de nacimiento de Cristo, así como para servir de punto de partida en la determinación de la epacta (con base en el procedimiento de los números radicales que describe Zamorano). Luego comenta la forma de calcular la epacta en el año de la muerte de Cristo (que según Sánchez de Cozar corresponde al año 35 d. C.) y lo mismo hace en cada uno de los cuatro años siguientes a ese.

A continuación pasa a describir una serie de tablas (pág. $87,87 \mathrm{v}$ y 88 ) que permiten determinar la epacta ajustada a la reformación del autor (esto es, no considerar bisiestos cada ciertos años separados por 144 años) y las cuales permiten, con el conocimiento del número áureo de un cierto año y los números radicales dados por el pulgar correspondiente, determinar directamente la epacta de ese año. Llama la atención, entonces, que Sánchez de Cozar elabore un número no despreciable de pulgares de la mano izquierda con distintos números radicales, los que hace necesarios para tener en cuenta aquellos años en los que opera la "simulación del bisiesto".

Posteriormente, y frente a la crítica de "cierto sujeto con presunciones de grande astrólogo" — que debió objetar de cómo un humilde cura en una aislada colonia española llegó a encontrar una solución al problema del calendario, superior a la que llegaron varios sabios europeos en 1582- defiende su posición de dos maneras: la una, señalando la voluntad de Dios de decidir a quién reparte los descubrimientos e invenciones particulares en los "tiempos que ha visto que ha convenido"; la otra forma es continuar con su crítica a la reforma gregoriana señalando lo que a su juicio fueron las omisiones de tales reformadores y reiterando que la duración del año no puede ser otra que $365^{d} 5^{h} 50^{m} 0^{s}$, así como asignar años bisiestos "simulados" cada 144 años a partir del $68 \mathrm{~d}$. C.

El capítulo 3 explica algunos detalles simples de cómo calcular los tiempos de la ocurrencia, tanto la luna nueva como la luna llena que el autor utilizó para construir las tablas de efemérides que están contenidas en el capítulo 5. Presenta dos ejemplos de cómo proceder para el cálculo de la luna nueva: para marzo (el cual él, siguiendo a Zamorano, considera aquí como el primer mes del año) y diciembre de 1681 (un año después de 1680, que fue bisiesto); la luna llena la calcula simplemente contando 15 días después de verificada la fase nueva.

La forma de determinar el ciclo solar, la letra dominical y la indicción para un año en particular se explica en el capítulo 4. Para las dos primeras lo hace con base en el uso de las tablas "perpetuas" contenidas en el capítulo 9 del segundo tratado (ver pág. 59v), una de cuyas columnas (la quinta) reproduce al inicio del capítulo (ver pág. 91v). Ex- 
plica el uso de tales columnas con base en el año en el que se desea calcular el ciclo solar y la letra dominical e incluye un ejemplo de su uso. Finiquita el capítulo explicando el ciclo de la indicción, que está conformado por 15 años, y comenta cómo ha de determinarse.

El capítulo 5 contiene una explicación detallada de las tablas de fases lunares - conjunción (luna nueva) y oposición (luna llena) - consignadas de mes a mes y que se muestran al final de dicho capítulo. Las tablas se inician desde el año 1675 y se extienden hasta el año 1736 (ver pág. 96 a 111), en total 62 años. Para cada año se especifica el mes, la fase, el día, la hora y el minuto en que ocurre el fenómeno (en tiempo contado desde el medio día) así como el número de grados de la constelación zodiacal correspondiente y el nombre de esta en el que se verifica el fenómeno (Figura 5).

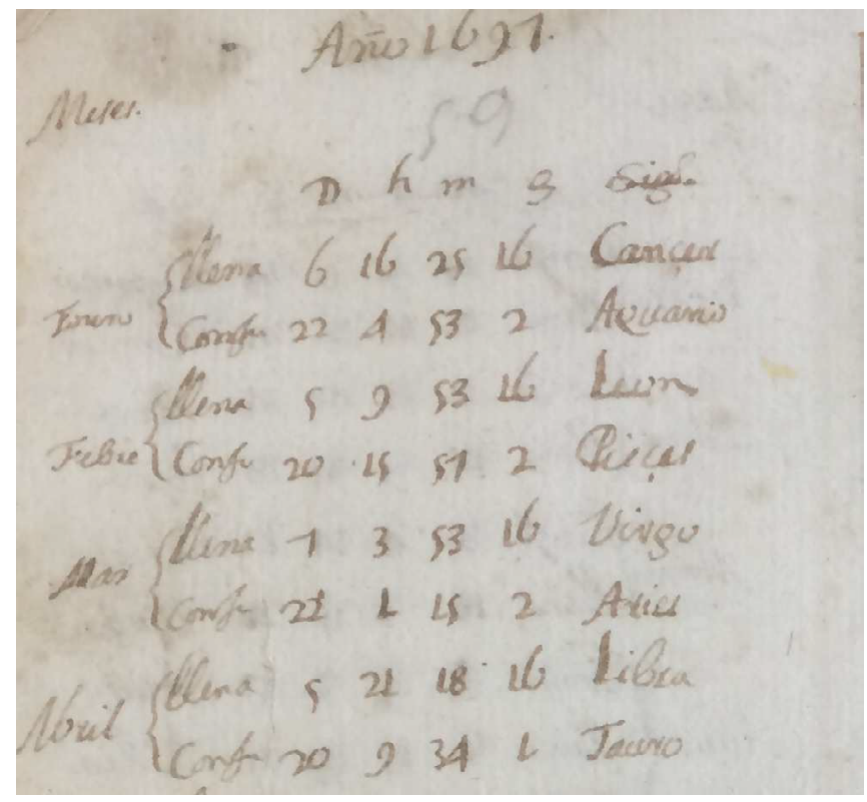

Figura 5: Parte de la tabla izquierda de la pág. 101v en la que se muestran los instantes (en día, hora y minuto) de las conjunciones y oposiciones de la Luna calculados para la hora local de Vélez así como su posición (en grados) dentro del signo zodiacal en que se verifica el fenómeno. Específicamente se muestran los primeros cuatro meses del año 1697

En la parte inferior de cada tabla anual, se especifican el número áureo, la epacta, el ciclo solar, la indicción, la letra dominical y la fecha de la Pascua de resurrección. Explica la utilización de los datos con un ejemplo (marzo de 1675) y de cómo se ha de proceder para determinar los tiempos contados de medianoche a medianoche, "que es el uso ordinario de la iglesia". Más adelante explica "el modo de perpetuar dichas 62 tablas siguientes de 62 en 62 años", de manera que con la información contenida en esas tablas se puedan predecir las demás fechas de esas fases lunares para años posteriores a 1736. Así que establece una serie de modificaciones, dependiendo de si los años que siguen al cabo del ciclo de 62 son bisiestos, o siguen a un año, o dos o tres de un bisiesto, para fijar los días y los tiempos correctos de tales fases.

El capítulo siguiente, el 6 , no es más que una simple extensión del anterior, ya que lo que contempla es hacer explícita las indicaciones ya vistas de cómo realizar una serie de modificaciones para extender el uso de las 62 tablas (de los años 1675 a 1736) y aplicarlas a los años inmediatamente siguientes y con ello hacerlas "perpetuas". De modo que incluye cuatro tablas adicionales con el mismo formato de las tablas del capítulo 5 pero correspondientes a los años 1737 a 1740 .

En el capítulo 7 el lector se entera de que los tiempos de las conjunciones y oposiciones que se hallan contenidos en los dos capítulos previos están referidos al meridiano de referencia de la ciudad de Vélez (municipio del actual departamento de Santander). Así que en el caso de que alguien, ubicado en otro lugar geográfico, desee conocer los tiempos en que ocurren tales fenómenos ha de remitirse a las tablas que el autor consigna al final de ese capítulo en el que se muestran las longitudes en unidades de tiempo (horas y minutos) con la indicación de si el lugar está al este del meridiano de referencia (en cuyo caso utiliza la letra a, que significa añadir ese valor a la hora dada en la tabla) o al oeste (utilizando la letra e, que significa restar). El autor adjunta dos ejemplos que contemplan ambos casos, tanto para un observador situado en Lima (Perú) como en Madrid (España).

Estas tablas (que comprenden las páginas 114 a 115v) presentan los nombres de las ciudades y, frente a ellas, en la primera columna, la respectiva latitud en grados (que el autor denomina "grados de altura de los polos"); la segunda y tercera columnas contienen la longitud en horas y en minutos y la cuarta columna la indicación ya vista de si está ubicada al este o al oeste del meridiano de Vélez. La tabla divide en varios sectores las respectivas ciudades, de manera que diferencia 35 ciudades correspondientes al Nuevo Reino de Granada y tierra firme, aunque Cartagena, Santa Marta y otros accidentes geográficos de la actual Colombia están en el sector correspondiente a "puertos de tierra firme". El autor consigna un número importante de poblaciones pertenecientes al dominio español, desde México hasta el Perú, incluyendo islas del mar Caribe, de Canarias y numerosas poblaciones de España.

De forma interesante, el capítulo 8 está redactado con una letra un poco más estilizada y adornada, la escritura es nivelada y es evidente que la pluma con que se hizo la escritura es un tanto más fina; a pesar de estos cambios, es indudable que su redactor continua siendo Sánchez de Cozar. Es posible, como explicación, que este capítulo haya sido redactado en un tiempo significativamente distinto al del resto del documento. Sea como haya sido, el autor explica inicialmente cómo los eclipses se repiten cada 18 años y diez u 11 días, esto es, el ciclo de Saros (aunque no lo menciona con ese nombre). Da una serie de pautas para calcular la fecha de los eclipses, explica el concepto de magnitud de un eclipse 
(qué tanto de la superficie de la Luna o del Sol se ven eclipsados) en términos del concepto de dígitos.

Ofrece un ejemplo de cómo se va repitiendo un eclipse de Luna a lo largo de los años: el 11 de enero de 1675, luego el 21 de enero de 1693 y luego el del 2 de febrero de 1711. Alude a 62 tablas de eclipses, con lo que quiere decir que presentará los eclipses a lo largo de 62 años comprendidos entre 1675 a 1736 (el mismo intervalo de tiempo con que presentó las conjunciones y oposiciones de la Luna en el capítulo 7). También ofrece una serie de indicaciones "para que con toda facilidad se puedan ir anotando perpetuamente los eclipses de 62 en 62 años". Al final de la explicación presenta, iniciando a partir del año 1675 , los eclipses de Luna y de Sol (ver pág. 118). En cada caso menciona el día y la hora (tiempo de Vélez), el tipo de eclipse, su magnitud, su duración y a cuántos grados de la constelación zodiacal se presenta el fenómeno. Infortunadamente, el documento en la actualidad finaliza en la pág. 123v, en la que alcanza a incluir los eclipses hasta el año 1700. Puesto que el autor mencionó que incluiría los eclipses hasta el año 1736, se desprende que un buen número de hojas correspondientes a este capítulo se perdieron. Como se recordará, el autor alude a un noveno capítulo en este tratado, el cual, por supuesto, también se perdió.

\section{Análisis}

Varios son los elementos que hay que destacar del contenido del manuscrito y que revelan aspectos de la naturaleza y los conocimientos que llegó a tener Sánchez de Cozar sobre astronomía y cronología. Para el caso probable de que el escrito se constituya en una medida apropiada de la extensión de sus conocimientos, hay que señalar que su erudición astronómica, aunque no era desdeñable, tampoco era profunda. Conocía aspectos fundamentales del modelo geocéntrico, diferenciaba los diferentes movimientos del Sol y de la Luna, y muy posiblemente identificaba los planetas y diferenciaba las constelaciones. Pero hay sutiles anotaciones y omisiones dentro del manuscrito que nos indican que no era propiamente un astrónomo avezado (y él mismo lo reconocía, pág. 25): era más del tipo de la comprensión teórica que de hacer las observaciones nocturnas correspondientes; no existe el registro de un solo nombre de una estrella (a excepción de la polar); tampoco hay algún registro o comentario de que sus predicciones sobre el novilunio y el plenilunio eran correctas o no; y lo mismo puede decirse sobre sus predicciones de los eclipses.

Al parecer los únicos registros observacionales son sus observaciones de los cometas de 1681 y de 1682 , de los cuales hablaremos en breve. Al parecer, las limitaciones que tuvo en adquirir un conocimiento más amplio de la astronomía de su tiempo se debió a la carencia de bibliografía más técnica al respecto, ya que sus libros de cabecera no ampliaban lo suficiente en esa materia. En ningún lado se hace alusión a que conociera la existencia del telescopio o algún otro instrumento astronómico salvo al astrolabio (pág. 89) del que atribuye su invención a Ptolomeo y lo describe como "maravilla poco entendida de muchos". No puede descartarse que poseyera uno de tales aparatos e incluso, que lo haya utilizado para determinar la latitud geográfica.

En lo que respecta a cronología, Sánchez de Cozar demuestra conocimientos no despreciables. Por un lado, dominaba la problemática surgida al querer acoplar los periodos tanto del año trópico como del mes sinódico conducentes a satisfacer los requerimientos para la determinación de la Pascua de resurrección. También dominaba con fluidez los distintos ciclos que permiten la determinación de dicha fiesta y era muy consciente de la dificultad que entraña establecer las fechas en que ocurrieron ciertos eventos a lo largo de la historia. Se nota que le dedicó grandes esfuerzos a su comprensión y, lo que es más importante, juzgó, aunque equivocadamente, que tenía mucho que aportar en la búsqueda de un correcto calendario para las necesidades de carácter religioso.

Pasamos a continuación a describir varios aspectos notables que, a nuestro juicio, merecen destacarse del contenido del manuscrito.

Primero. Una concepción dinámica de los cielos, enmarcada dentro del modelo geocéntrico, que lo diferencia de los filósofos, teólogos y astrónomos escolásticos que lo han precedido, en el sentido de que ofrece una explicación del movimiento de los cielos de naturaleza sorprendentemente física. Hasta donde se ha podido indagar, la concepción de tal modelo parece ser original de Sánchez de Cozar. El autor no requiere de "inteligencias angélicas" para dotar de movimiento a los cielos ni tampoco del "primer motor" aristotélico (pág. 35). En su lugar propone la existencia del punto céntrico de la gravedad (PCG), el cual es un "punto estable indivisible fundamento de la estabilidad de todo peso igual" el cual se halla "en el medio y semidiámetro de los dos cuerpos tierra y agua el cual es el fundamento de ser la fábrica del mundo redonda y el fundamento del movimiento circular de las esferas" (pág. 13v).

Esto quiere decir que el PCG no solo es responsable del movimiento circular de los cielos si no que también hay que atribuirle la forma circular de los mismos. Una de las razones por las que el Empíreo permanece inmóvil es que toda su estructura está "unidamente de peso natural al punto céntrico de la gravedad", lo que puede interpretarse como que la esfera que constituye el Empíreo es perfectamente simétrica y que al ser atraída en todas sus partes por el PCG no puede existir mayor atracción en un punto que en otro (pues están a igual distancia) y de ahí su inmovilidad perfecta. En contraste, los planetas, y el cielo estrellado son asimétricos en el sentido de que constan de un ñudo en el que está encajado el planeta propiamente dicho (y en el caso del cielo estrellado, el punto de Aries) y, en consecuencia, están "desquiciados", es decir, se encuentran en una situación de desequilibrio permanente, cada uno con distinta velocidad, debido a la atracción del PCG.

El movimiento de estos planetas es de occidente a orien- 
te con respecto al cielo estrellado, esto es, contrario al movimiento de "rapto" (es decir, el movimiento aparente de los cuerpos celestes de oriente a occidente y que es debido a la rotación de la Tierra de occidente a oriente, denominado en el presente como movimiento diurno). Además, el autor propone que las pirámides de los cielos rozan en ocasiones con los cielos inmediatamente anexos (como lo hace el "ñudo" de Aries con el cielo de Saturno), lo que permite una interacción entre ellos que explica por qué ciertos planetas parecen detenerse o retrogradar en su movimiento de occidente a oriente con relación al cielo estrellado; añade un comentario interesante sobre la dinámica relativa entre los cielos: "el pararse de los cielos [...] no es otra cosa que caminar a la par con el estrellado; y el retroceder no es otra cosa que caminar más veloz que [...] al movimiento del rapto" (pág. 29v).

El caso de la justificación de la inmovilidad de la Tierra es más complicado. Sánchez de Cozar admite que la Tierra no es una esfera perfecta, pues frente a la diversidad de valores del radio propuesto por varios autores manifiesta que "todos dicen verdad" y secunda la hipótesis de Juan Pérez de Moya de que la "Tierra está más elevada hacia la región media que por otra"; la consecuencia lógica de esto es que el centro de la Tierra difiere del punto céntrico de la gravedad (pág. 39v). Pero, para no incurrir en contradicciones, sus argumentos para asignar a la Tierra completa inmovilidad son fundamentalmente de autoridad, para lo cual cita dos salmos de David.

La concepción del movimiento de los cielos que propone Sánchez de Cozar sorprende por su originalidad y por estar dotada de cierto tinte de naturaleza física. Aun cuando existe un enorme abismo entre esta concepción y la forma como Isaac Newton construyó la física tal y como la conocemos, no puede uno dejar de preguntarse si el neogranadino llegó a conocer, así fuera de oídas, ciertos aspectos del pensamiento newtoniano. La posibilidad, aunque excesivamente remota, existe, pues la primera edición de los Principios Matemáticos de Filosofía Natural es de 1686, esto es, una década antes de la redacción del documento que nos ocupa.

Segundo. La creación de un nuevo cielo existente entre Mercurio y la Luna (pág. 7v, 15 y 15v). Sánchez de Cozar fue sensiblemente impactado por la aparición del gran cometa de 1680 (el que observó a inicios de 1681), a lo que siguió la aparición de otro “pequeño" en el año de 1682, para los cuales interpretó como augurios en favor del cristianismo ante su lucha contra el Islam. Puesto que se trata del primer registro de la aparición de cometas realizado por un criollo en la Nueva Granada, ampliaremos la información sobre estas observaciones. El impacto de la aparición del primero - "aquel tan espantoso como nunca visto cometa"- fue tan mayúsculo que el sacerdote propuso la existencia de un nuevo cielo al que denominó el incógnito "por no haber sido conocido hasta aquí" (pág. 24v).

El cometa que observó a inicios de 1681 fue el cometa
Kirch, denominado también gran cometa de 1680, cometa de Newton y, modernamente, como C/1660 V1. Este cometa, que fue el primero que se descubrió por telescopio, fue extensamente observado en Europa por renombrados astrónomos y es considerado uno de los más brillantes y notorios de todos cuanto se vieron durante el siglo XVII. Sus elementos orbitales pueden observarse en la tabla 1 .

El cometa Kirch, desde su descubrimiento a mediados de noviembre y hasta mediados del mes siguiente, fue observado en la madrugada; al pasar por el perihelio comenzó a observarse al anochecer, es decir, después de ocultarse el Sol. Si bien es cierto que el cometa ya era fácilmente observable hacia el horizonte occidental desde los últimos días de diciembre de 1680, Sánchez de Cozar solo comenzó a observarlo pocos días después: "desde 4 de enero en que se apareció hasta 15 de febrero en que feneció" (pág. 25). Aunque el sacerdote no lo menciona, durante ese tiempo el cometa fue visto atravesando las constelaciones de Pegaso, Andrómeda, Piscis, Triángulo y Perseo.

Tabla 1. Elementos orbitales del cometa C/1660 V1 tomados de Kronk (1999), pág. 373. Las coordenadas angulares están referidas al equinoccio medio y eclíptica del 2000.0.

\begin{tabular}{ll}
\hline Elemento & Valor \\
\hline Distancia pericéntrica $(q)$ & 0.006222 (u.a.) \\
Excentricidad $(e)$ & 0.999986 \\
Inclinación $(i)$ & $60.6784^{\circ}$ \\
Longitud del nodo ascendente $(\Omega)$ & $276.6339^{\circ}$ \\
Argumento de lat. del per. $(\omega)$ & $350.6128^{\circ}$ \\
Tiempo del paso por el per. $\left(t_{0}\right)$ & 18.4876 dic. 1680 \\
\hline
\end{tabular}

Sánchez de Cozar, como ya dijimos, y con base en la observación de este cometa, propone la existencia de un cielo adicional entre Mercurio y la Luna. Dos son las razones para llegar a esa conclusión: la primera es que vio "pasar la Luna por debajo de él, por dos veces (precediéndole Mercurio)" y la segunda, por su velocidad con respecto a las estrellas, la cual estimó, como veremos más adelante. En relación con la primera razón, e independiente del raciocinio que le condujo a pensar que la Luna cruzó por debajo de la cola del cometa, una simulación del cielo para esas fechas (realizada con el programa Voyager 4 de la empresa Carina Software) permite establecer que, si vio la Luna cruzar por la cola del cometa, debió ser unos pocos días en que la Luna se ubicaba más al este que el mismo cometa, lo cual es factible, dado los reportes que asignaron una extensión de la cola comprendida entre unos 60-70 ${ }^{\circ}$ de longitud (Kronk (1999)); si ese fue el caso, solo pudo observar el cruce en una ocasión, lo cual posiblemente ocurrió a finales del mes de enero. Es de advertir que para el tiempo que observó el cometa, éste se desplazó paralelamente a la eclíptica y en la dirección en que se desplazan los planetas: de occidente a oriente. 
Resulta interesante que Sánchez de Cozar estimó la tasa diaria de desplazamiento del cometa: $1^{\circ} 12^{\prime}$. Con eso parece no advertir que el movimiento del cometa a través del cielo no es constante: en los primeros días de enero era cercano a los $4^{\circ}$; solo hasta finales de ese mes cayó a los niveles que él anotó. Con esa estimación calcula el periodo asumiendo que el objeto es periódico y obtiene: $360^{\circ} / 1^{\circ} 12^{\prime}=300$ días. Este es, pues, el valor que asigna como periodo al cielo "incógnito" (pág. 36). Nótese que tal valor es intermedio al del Sol y el de la Luna, pues recuérdese que para Sánchez de Cozar (como para Zamorano) el periodo de Mercurio es igual al del Sol.

El cometa "pequeño" que Sánchez de Cozar dice haber observado en el año de 1682 fue el cometa Halley (1P/1682 Q1). Su aparición en esa ocasión no fue tan notoria, pues apenas le dedicó un fugaz comentario: "se nos apareció [...] en forma de cetro en el signo de Acuario del estrellado" (pág. 25). Los elementos orbitales correspondientes a ese paso del Halley están contenidos en la tabla 2.

Tabla 2. Elementos orbitales del cometa 1P/1682 Q1(Halley) tomados de Yeomans (1977). Las coordenadas angulares están referidas al equinoccio medio y eclíptica de 1950.0.

\begin{tabular}{ll}
\hline Elemento & Valor \\
\hline Distancia pericéntrica $(q)$ & 0.582608 (u.a.) \\
Excentricidad $(e)$ & 0.967923 \\
Inclinación $(i)$ & $162.2657^{\circ}$ \\
Longitud del nodo ascendente $(\Omega)$ & $54.8522^{\circ}$ \\
Argumento de lat. del per. $(\omega)$ & $109.2054^{\circ}$ \\
Tiempo del paso por el per. $\left(t_{0}\right)$ & 15.2807 sep. 1682 \\
\hline
\end{tabular}

Una simulación de la aparición del cometa Halley en ese año permite verificar que comenzó a ser visto a finales del mes de agosto en horas de la madrugada, lo que es coherente con lo consignado por Kronk (1999). En esos días el cometa se desplazó a través de las constelaciones del Lince y Leo Menor; a principios de septiembre ya era visible al atardecer y se movió a través de las constelaciones de Cabellera de Berenice, Virgo y Libra, donde desapareció a finales de la tercera semana de septiembre. En esta visita la visibilidad de este célebre cometa periódico fue muy discreta. Pero no se entiende por qué Sánchez de Cozar escribió que lo vio en Acuario, a menos que lo haya confundido con el cometa de Kirch que, en los primeros días de enero, como ya dijimos, se desplazó por Pegaso, que está próximo a Acuario. La memoria pudo haberle jugado una mala pasada, pues al momento de redactar esas líneas ya habían pasado unos tres lustros desde la aparición de tales cometas.

Debido a la pérdida de varias hojas al final del tercer tratado, en el cual Sánchez de Cozar incluyó un capítulo sobre los cometas, no nos es posible tener un conocimiento más acertado de lo que él opinaba sobre estos fenómenos celestes. Pero es evidente que, a diferencia de la postura escolástica, los ubicaba más allá del cielo de la Luna, esto es, como planetas propiamente dichos; pero no es claro si él era consciente de la variabilidad no solo del brillo sino de la longitud o dirección de la cola de los cometas. Sin embargo, hay que reconocer que si bien adjudica a los cielos la propiedad de "incorruptibilidad", la palabra "inmutabilidad" o alguno de sus sinónimos no aparece explícitamente en el escrito, por lo que es factible que admitiera algún tipo de cambio en los cielos. De hecho, implícitamente lo asume, pues afirmó que "y es sin duda haber sido nunca visto este cometa del cielo incógnito, pues juzgo no se hallará autor de esta ciencia por antiguo que sea que dé razón de haber sido visto", de lo que se infiere que necesariamente debe asumir la existencia del cambio de los cielos, esta vez, con la aparición de un objeto nunca antes observado que está entre Mercurio y la Luna.

Es apenas evidente que la introducción del cielo "incógnito" no es muy coherente y genera toda una serie de preguntas sobre la consistencia de la propuesta: ¿Es el incógnito un repositorio de cometas o lugar de uno solo? Si es lo segundo, ¿por qué no surge el cometa aproximadamente cada 300 días? Como es evidente que ello no ocurre, ¿qué mecanismo propone para que el incógnito solo se manifieste en determinadas circunstancias? Al margen de los vacíos e incoherencias de la propuesta, lo meritorio del autor es arriesgarse a proponer una hipótesis. Si los antiguos aventuraron la existencia de toda una serie de cielos, algunos de los cuales él considera innecesarios, ¿qué impide que él proponga uno de su propia factura?

Tercero. Cronología y reforma del calendario. Un aspecto llamativo del autor consiste en asegurar que el calendario gregoriano adolece de deficiencias y que es susceptible de mejorarse, no solo para satisfacer efectivamente lo establecido por el concilio de Nicea, para efectos de celebrar en la fecha precisa la Pascua de resurrección, sino además lograr una debida coherencia cronológica con los sucesos relatados en la Biblia. En particular, y a lo largo del manuscrito (pág. $11,55,62,62 \mathrm{v}, 68 \mathrm{v}, 72,79 \mathrm{v}, 84$ y 84v), hace énfasis en el evento "milagroso" ocurrido al momento de la muerte de Cristo cuando, de acuerdo con lo relatado por el evangelista Lucas (23: 44-45), se verificó un oscurecimiento del Sol que duró tres horas sobre toda la Tierra. Esto comúnmente se ha interpretado como la observación de un eclipse de Sol. Sánchez de Cozar también cita a Dionisio el Aeropagita, quien en su libro "De los nombres divinos", en la epístola VII, asegura haber sido testigo de ese eclipse.

Sánchez de Cozar considera verdad inamovible el suceso de tal eclipse, aunque es uno ciertamente anormal, pues sabe bien que un eclipse total de Sol no puede tener una duración tan elevada ni que se pueda verificar a lo largo y ancho del planeta. En todo caso, tal tipo de eclipse solo puede presentarse, como es apenas evidente, en la conjunción de la Luna con el Sol. Pero aquí hay un problema, pues si Cristo murió al día siguiente de conmemorarse la Pascua judía, la que se supone se verifica en luna llena, esto es, en plenilu- 
nio, la Luna no pudo ser ocultada por el Sol al momento de la muerte de Cristo, a menos que haya habido una intervención divina que haya desplazado la Luna casi ciento setenta grados de su posición. Por supuesto, Sánchez de Cozar no duda de que haya sido así, por lo que considera que es indispensable tener en cuenta en los cálculos cronológicos ese desplazamiento milagroso de la Luna, pues ciclos como el del número áureo y la epacta lógicamente han de ser modificados a partir de tal evento.

De modo que el sacerdote debió escoger como fecha de muerte de Cristo el año 3855 después de la creación del mundo porque la epacta de tal año es igual a 4 , lo que permite tener para el 25 de marzo (es decir, en la muerte de Cristo) luna nueva y la letra dominical corresponde a la letra B. Fijado esto, y como la tradición cristiana sostiene que Cristo vivió 33 años, determina como fecha de nacimiento de este el año 3821 después de la creación del mundo (que en la escala de tiempo que operamos corresponde, por supuesto, al año $1 \mathrm{~d}$. C.), año cuya letra dominical es la letra B, por lo que el 25 de diciembre cae en domingo.

De igual forma, Sánchez de Cozar, por razones que no son del todo claras, fija como duración del año medio unos 44 segundos más largo que la duración del año adoptada por los asesores de Gregorio XIII. Recordemos que el valor del año trópico es de $365^{d} 05^{h} 48^{m} 45.19^{s}$ (Seidelmann, 1992), esto es, 31 segundos más corto que la duración del año que adoptó Gregorio XIII y 75 segundos más corto que el que adoptó Sánchez de Cozar. No hay nada que sugiera que el mismo sacerdote haya medido dicha duración; más bien lo hace con el propósito de asegurar cierta coherencia cronológica de lo relatado en la Biblia. La adopción de esa duración, como vimos, ocasiona que el calendario civil adelante en un día al año trópico cada 144 años, lo que explica su propuesta de hacer que cada vez que se llegue a este número de años se establezca un año normal de 365 días aunque sea divisible por 4 ("simular un bisiesto").

El propósito del sacerdote es asegurar la coherencia de los datos cronológicos referidos en la Biblia, fijar de una vez por todas la fecha de nacimiento de Cristo y, de paso, establecer unas normas que permitan fijar de forma correcta la fecha de la Pascua cristiana tal y como se estableció en el concilio de Nicea. Sánchez de Cozar critica la reforma gregoriana en el sentido de eliminar 3 días cada 400 años para que el calendario civil no se adelante en ese tiempo con respecto al año trópico; en su lugar propone, como ya hemos visto varias veces, que cada 144 años se establezcan años comunes de 365 días aunque sean divisibles por 4; algunos de esos años son, a partir del siglo XVIII y con respecto a la era cristiana: 1796, 1940, 2084, 2228, etc. También critica que la reforma gregoriana haya eliminado 10 días del mes de octubre de 1582; el sacerdote considera que debieron haberse eliminado 11 días, y no en el mes de octubre sino en el mes de marzo (ver pág. 79v). Juzga entonces que al año de 1582 debió asignársele por letra dominical $\mathrm{D}$ y no $\mathrm{C}$ (antes de la reforma le correspondía la G). Esto explica por qué las letras dominicales que se muestran, por ejemplo, en la tabla de la pág. $60 \mathrm{v}$ y las que existen en la parte inferior de las tablas de fases lunares, no sean iguales a las que se utilizan formalmente, lo que implica que en su reforma al calendario, por poner por caso, el 1 de enero de 1696, que cayó en domingo, para Sánchez de Cozar cayó un viernes.

Cuarto. Predicción de fases lunares y eclipses. Hasta donde sabemos, Sánchez de Cozar, es la primera persona existente en el Nuevo Reino de Granada que calculó efemérides astronómicas. Y no solo eso: lo hizo con relación a la hora local de una población del nuevo mundo (Vélez). El manuscrito contiene predicciones de los tiempos en que ocurren la luna llena y la luna nueva entre los años comprendidos entre 1675 y 1740; así mismo, establece en qué punto de la esfera celeste ocurre el fenómeno, pues indica el signo zodiacal y el número de grados dentro de él.

Otro tanto se puede decir con relación a las predicciones de eclipses de Luna y de Sol. También calculó los tiempos locales de los eclipses comprendidos entre 1675 y 1736 aunque, como ya se dijo, en la actualidad el manuscrito solo contiene los eclipses hasta el año de 1700.

Si bien es cierto que la forma empleada por Sánchez de Cozar para mostrar los tiempos de las fases lunares así como los eclipses lunisolares tienen virtualmente idéntica presentación y descripción a como aparecen en el libro de Zamorano, el primero no pudo copiarlas del segundo por una sencilla razón: la edición de 1594 que, presumiblemente, fue la que consultó el sacerdote, contiene las fases de la Luna entre 1594 y 1654 y eclipses lunares entre 1584 y 1606 . Igualmente, la edición de 1621 del libro de Zamorano contiene las fases de la Luna comprendidas solo entre 1620 y 1654 y eclipses lunares entre 1620 y 1630 . La consecuencia de esto es que Sánchez de Cozar debió calcular los plenilunios, novilunios y eclipses lunisolares y, por supuesto, debió constituirse en una tarea que le demandó bastante tiempo y esfuerzo.

Quinto. Geografía neogranadina. Como ya se dijo, en el capítulo 7 del Tercer Tratado Sánchez de Cozar presenta varias tablas con valores de latitudes y longitudes (en tiempo) de numerosas poblaciones de España y sus colonias. Al comparar estos valores con los existentes en el capítulo 63 del libro de Zamorano (Zamorano, 1594) se evidencia que el sacerdote, en su gran mayoría, los tomó de allí, advirtiendo que los valores de longitud están referidos a la población de Vélez y no a Sevilla, que es como lo referencia el español. Sin embargo, hay diferencias. La principal es que Sánchez de Cozar construye una tabla adicional titulada "Nuevo Reino de Granada y Tierra Firme" inexistente en el libro de Zamorano (Figura 6). La tabla que elabora el sacerdote muestra la latitud y longitud de 35 poblaciones y lugares geográficos del interior del Virreinato de la Nueva Granada (las ciudades costeras colombianas como Cartagena y Santa Marta están en otra tabla que tituló "Puertos de Tierra Firme", tal y como lo hizo Zamorano).

En la obra de este último, las poblaciones de Bogotá, 
Cali, Panamá, Popayán, San Juan, Timaná y Villaviciosa aparecen haciendo parte de una tabla que lleva por título "Pueblos de la mar del Sur y del Piru". En la tabla de Sánchez de Cozar sobre las poblaciones del Nuevo Reino de Granada incluye, aparte de las siete que incluye Zamorano, otras 28; la mayoría son poblaciones de fácil identificación, tales como (por citar algunas): Vélez, Buga, Muzo, Mariquita, Mérida, Pamplona, Anserma, Girón, Sogamoso, Tocaima, Villa de Leiva y San Gil; otras, por el contrario, no lo son tanto como Palma, San Francisco de la Concha y Trugillo.

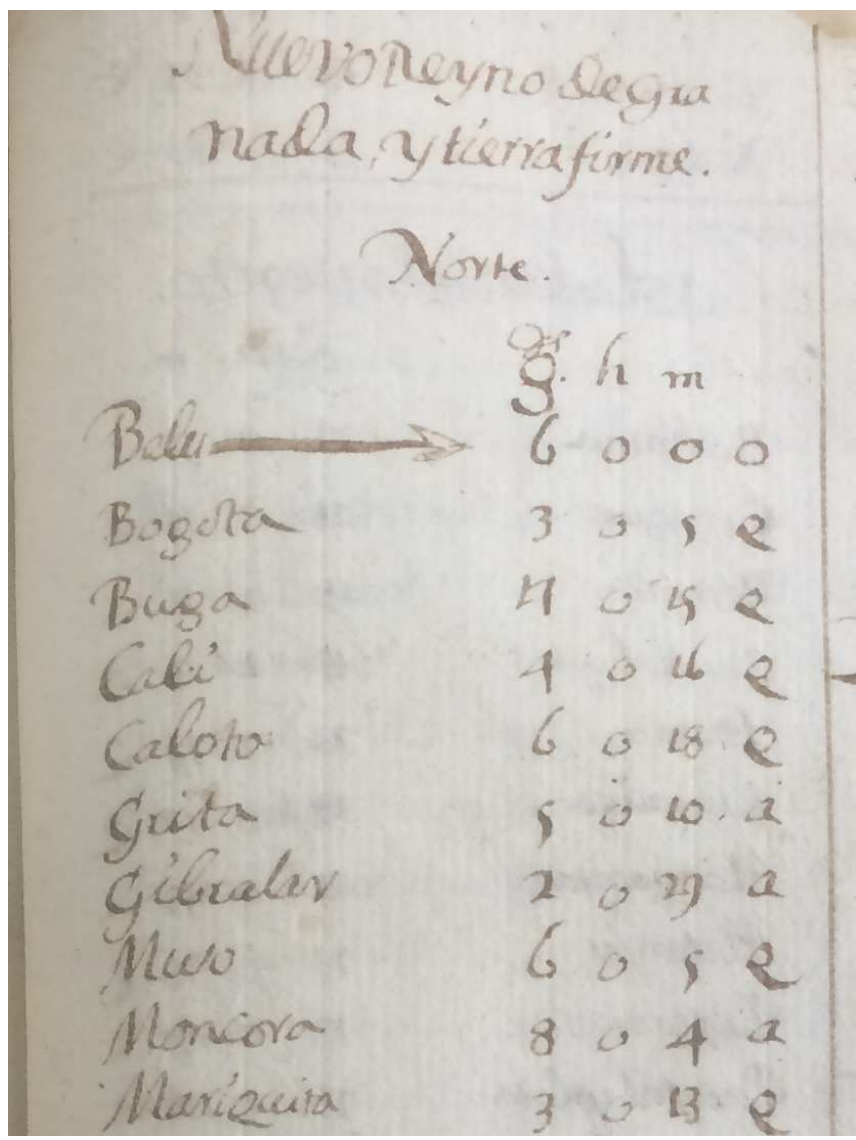

Figura 6: Parte de la tabla izquierda de la pág. 114, en la que consigna la posición geográfica de varias poblaciones del Nuevo Reino de Granada.

Sánchez de Cozar, como Zamorano, expresa las latitudes solo en grados (en número entero) y la longitud en unidades de tiempo (en horas y minutos). Para determinar las longitudes de las poblaciones respecto a Vélez $(\lambda)$ con base en la longitud dada por Zamorano $\left(\lambda_{Z}\right)$ utilizó la expresión: $\lambda=\lambda_{Z}-4^{h} 47^{m}$, donde este último valor representa la longitud de Vélez con respecto a Sevilla (el valor correcto es $4^{h} 31^{m}$ hacia el oeste). Si $\lambda>0$, la longitud es hacia el oeste de Vélez, que Sánchez de Cozar especifica con la letra e; si $\lambda<0$, la longitud es hacia el este, lo que especifica con la letra a.
Si bien es cierto que la mayoría de los valores que Sánchez de Cozar registró son idénticos (o pueden inferirse directamente, como las longitudes) a los que aparecen en el libro de Zamorano hay, sin embargo, algunas diferencias. Puede verse que modificó en una unidad (bien arriba o hacia abajo) las latitudes de Bogotá, Popayán y Cartagena; de igual forma aparecen algunas diferencias en las longitudes de Popayán y Villaviciosa. Casi todos esos cambios apuntan hacia los valores correctos; sin embargo, no se conoce la fuente que Sánchez de Cozar utilizó para modificar algunos de los valores de las coordenadas y, más significativo aun, aquellos valores de latitudes y longitudes de las restantes 28 poblaciones que no aparecen en el libro de Zamorano. No se descarta que haya utilizado distintas fuentes y alternativas para hacerlo, como, por ejemplo, haber interpolado las coordenadas a partir de un mapa. Una posibilidad, algo remota, es que él mismo haya derivado varias, en particular las cercanas a su lugar de residencia, tales como Girón, San Gil, Villa de Leyva, Valle del Santo Ecce Homo y Tunja, las que pudo determinar o bien astronómicamente o con base en el conocimiento algo aproximado de la distancia topográfica entre ellas.

Dejando a un lado los errores en los que Sánchez de Cozar incurrió en la asignación de esas coordenadas, ciertamente excusables, hasta donde sabemos la tabla en referencia se constituye en la más antigua información relacionada del conocimiento geográfico de la nación realizado por un criollo; que los haya derivado de un mapa o los haya obtenido de la observación astronómica es algo que está por verse. Pero su intención de establecer una ciudad neogranadina como referencia para las longitudes de las demás poblaciones del planeta, aunado a su deseo de que el libro fuera publicado por el Rey de España y, en consecuencia, conocido por el mundo, manifiesta un anhelo de empoderamiento y posicionamiento de la nación en la que vive, intención que antecede por más de un siglo a la que hizo Francisco José de Caldas en su adecuación y elaboración de mapas con referencia o bien a Cartagena de Indias o a Santafé de Bogotá (Portilla (2019)).

Sexto. Descripción del clima en la zona que habita. Como se vio en el capítulo 17 del segundo tratado, Sánchez de Cozar contrasta los intervalos en los que se verifican las épocas de lluvia y de verano aquí en el trópico con lo que sucede en España. El sacerdote escribe: "porque el tiempo que en toda España se dice verano por caliente y húmedo [...] (comenzando desde 21 de marzo) le decimos nosotros invierno por muy lluvioso, cual nos comienza desde 22 de febrero con los mismos efectos de reverdecer las plantas". Con relación al verano (estío) y el otoño, señala: "casi no difieren en sus efectos aunque difieren en sus entradas: pues entrando el estío en España a 21 de junio y el otoño a 21 de septiembre, entran en estas partes a 24 de mayo ya 24 de agosto. Y el invierno que en España entra a 21 de diciembre difiere en el temperamento y en la entrada más no en los efectos: porque si en España pela y deshoja las plantas por 
frío y húmedo y de muchas aguas, acá hace lo mismo por caliente y seco y de muchos soles, desde 23 de noviembre hasta 21 de febrero". Sin embargo, advierte que tales intervalos no se siguen estrictamente, "pues en unos años se anticipan y en otros se posponen y en otros no hay más de un verano y un invierno". Esta descripción del clima a lo largo del año es la primera, hasta donde sabemos, que hace un criollo de una región específica de la Nueva Granada.

\section{Conclusiones}

El tratado de astronomía del sacerdote Sánchez de Cozar se constituye en un documento de extrema valía, cuya importancia en la historia del pensamiento en el país no se corresponde con la casi total indiferencia de la que ha sido objeto por parte de historiadores y astrónomos colombianos a partir de mediados del siglo XIX, cuando fue rescatado del olvido y dado a conocer fundamentalmente como obra literaria. La simple iniciativa de un habitante de Soatá, junto con el patriotismo de Vergara \& Vergara, que aseguró la conservación del manuscrito $-\mathrm{y}$ de documentos de otros autores - se constituyeron en algunos de los eventos que impidieron que el extenuante trabajo de un desconocido y humilde sacerdote descendiente de indígenas pasara al olvido. Por supuesto, el hecho de que Sánchez de Cozar hubiese redactado varias versiones de su manuscrito (ignoramos si realizó otras con posterioridad a 1696) aumentó la posibilidad de que alguna de ellas se conservara, lo que no ocurrió con numerosos otros escritos del periodo de la conquista y la colonia que el mismo Vergara \& Vergara refiere y que atribuye al "total desamor por los estudios históricos de la patria", dando a entender que casi fuera política de estado ejercer hostilidad hacia el "estudio y fomento de nuestra historia” (Vergara \& Vergara (1867), pág. xviii).

Aparte del hecho fortuito de que la obra de Sánchez de Cozar haya sobrevivido hasta nosotros y permitido, a través de ella, saber de la existencia de este sacerdote amante del saber, inquieto, persistente, dispuesto a proponer ideas distintas a las de otras corrientes más conformistas, es apenas justo afirmar que éste merece ser etiquetado como el primer pensador original que surgió en el Nuevo Reino de Granada y se adelantó en más de un siglo a Caldas en la redacción de las primeras efemérides astronómicas referidas a una población local. De hecho, la originalidad de pensamiento de Sánchez de Cozar - dejando a un lado su geocentrismo y escolasticismo - al proponer una explicación dinámica de los cielos, un nuevo cielo entre la Luna y Mercurio y una propuesta alternativa de reforma al calendario, señalan a alguien mucho más arriesgado en la innovación y la propuesta y más concentrado en la continuidad de su trabajo de lo que alguna vez llegó a ser el abogado payanés, para quien la astronomía no pasó de ser una mera herramienta subsidiaria de la geografía.

El manuscrito en cuestión es, pues, una muestra del trabajo y pensamiento independiente de un sacerdote neogranadino quien, en medio de las privaciones y obliga- ciones propias de su cargo, estaba convencido de su capacidad y originalidad de pensamiento, seguro de que podía ofrecer ideas alternativas al mundo, pues juzgaba su obra lo suficientemente coherente y original como para ser mandada a imprimir por el Rey de España y leída por el Papa. Jamás sobremos cuánto tiempo esperó una respuesta o si murió ansiosamente esperándola. Pero confiemos que la región geográfica en la que nació sepa por fin reconocer sus dotes y esfuerzos para que su obra "no padezca las cavernas del olvido" (pág. 8v).

\section{Agradecimientos}

Los autores agradecen a las parroquias de las poblaciones de Vélez y El Socorro (Santander) por facilitar la búsqueda de información en sus respectivos archivos para la elaboración de este trabajo. Igualmente, agradecen a varios funcionarios de la Biblioteca Nacional que permitieron el acceso para consultar en físico el manuscrito en cuestión. Por último, agradecen los comentarios del profesor Eduardo Brieva y de un árbitro anónimo que permitieron mejorar la redacción del artículo.

\section{Conficto de intereses}

Los autores declaran no tener ningún conflicto de intereses.

\section{Referencias}

De Pineda J. (1589). Primera parte de los treinta y cinco diálogos familiares de la agricultura cristiana, Imprenta de la casa de Pedro de Adursa y Diego López, Salamanca.

Del Rey J., Marquínez G. (2004). Breve tratado del cielo y los astros del M. Javeriano Mateo Mimbela (1663-1736), Pontificia Universidad Javeriana, Archivo Histórico Javeriano, Bogotá.

Kronk G. (1999). Cometography: A Catalog of Comets (Vol. 1: ancient-1799), Cambridge University Press, Cambridge.

Marquínez G., Del Rey J. (2005). Física especial y curiosa del maestro javeriano Francisco Javier Trías (1755), Pontificia Universidad Javeriana, Archivo Histórico Javeriano, Bogotá.

Marti J. (1991). Obras completas, volumen 21, cuadernos de apuntes, Editorial de ciencias sociales, La Habana.

Moreno F., Portilla J. G. \& Zitzmann E. (2018). El Astrolabio. 17: 60-74.

Pinzón R. (1993). Cuadernos de filosofía latinoamericana, 54-55: 155-167.

Portilla J. G. (2019). La astronomía en Francisco José de Caldas, en impresión.

Seidelmann K. (1992). Explanatory Supplement to the Astronomical Almanac, University Science Books, Mill Valley.

Vergara y Vergara J. M. (1867). Historia de la literatura en Nueva Granada, Imprenta de Echavarría hermanos, Bogotá.

Yeomans D. K. (1977). The Astronomical Journal, 82: 435-440.

Zamorano R. (1594). Cronología y repertorio de la razón de los tiempos, Imprenta de Rodrigo Cabrera, Sevilla. 